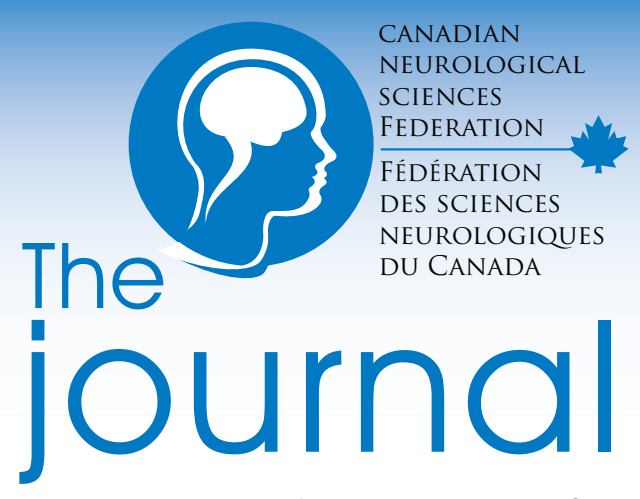

Canadian Journal of Neurological Sciences

Volume 39 Number 4 (Supplement 4) July 2012

\title{
Canadian Guidelines on Parkinson's Disease
}

$17 \frac{\text { Parkinson Society Canada }}{\text { Société Parkinson Canada }}$

A Peer-reviewed SUPPLEMENT to The Canadian Journal of Neurological Sciences

These guidelines are endorsed by the Canadian Neurological Sciences Federation 
https://doi.org/10.1017/S031716710001516X Published online by Cambridge University Press 


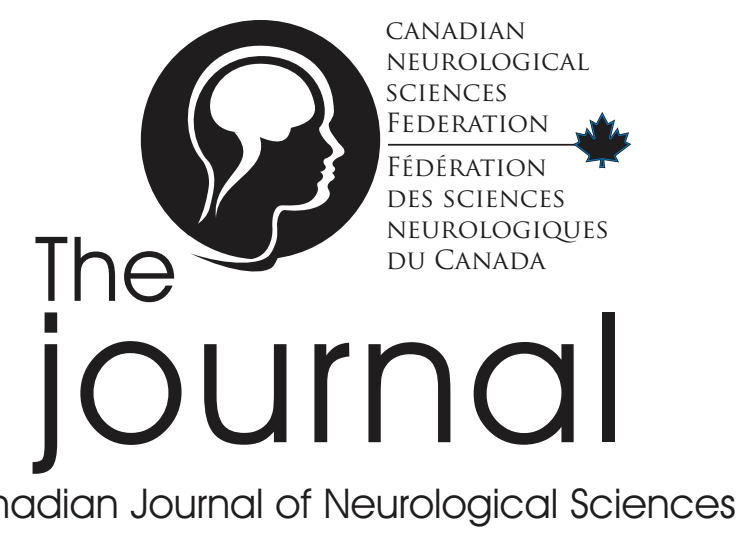

\section{$\begin{array}{llllll}\text { Volume } 39 & \text { Number } 4 & \text { S } & \text { Supplement } 4, & \text { July } 2012\end{array}$}

S1

S2

S5

S7

S10

S10

S11

S12

S14

S16

S17

S19

S22

S24
Canadian Guidelines on Parkinson's Disease Introduction

Guideline Development Process

Communication

Section 1

Diagnosis and Progression

Section 2

General Treatment Considerations

Section 3

Pharmacological Therapy for Motor Symptoms in Early PD Section $3 A$

Pharmacological Therapy for Motor Symptoms in Later PD Section $3 B$

Treatment - Surgery

Section $3 C$

Treatment - Other Treatment Options Section $3 D$

Non-Motor Features of PD - Mental Health

Section 4

Sleep Disorders

Section $4 B$

Autonomic Dysfunction Treatment - General Considerations Section $4 C$

Supplemental Tables

Summary of Canadian Recommendations with References 


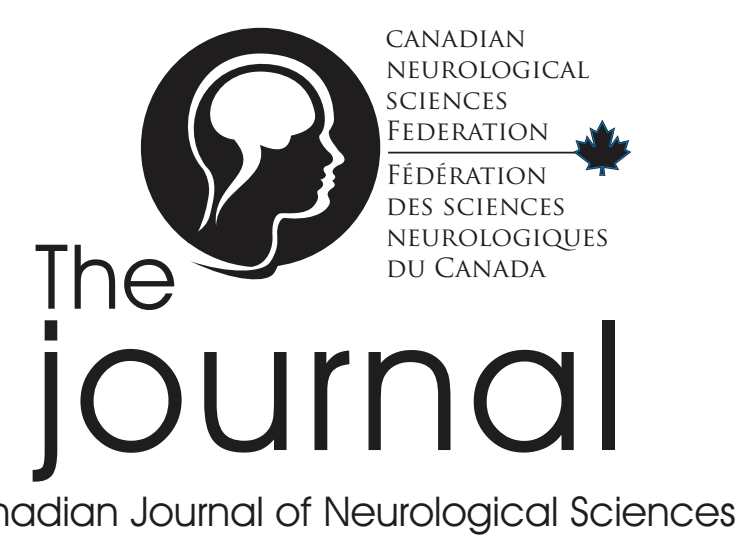

\section{$\begin{array}{lllllll}\text { Volume } 39 \quad / & \text { Number } 4 \quad / & \text { Supplement } 4 \quad / & \text { July } 2012\end{array}$}

Editor-in-Chief/Rédacteur en chef

G. Bryan Young LONDON, ON

Associate Editors/Rédacteurs associés

J. Max Findlay EDMONTON, AB

Timothy J. Benstead HALIFAX, NS

Mike Poulter LONDON, ON

Serge Gauthier VERDUN, QC

Robert Hammond LONDON, ON

Mary Connolly vancouver, BC

Past Editors/Anciens rédacteurs en chef

Douglas W. Zochodne CALGARY, AB

James A. Sharpe TORONTO, ON

Robert G. Lee CALGARY, AB

Robert T. Ross WINNIPEG, MB

(Emeritus Editor, Founding Editor)

\section{Editorial Board/Conseil d'éditorial}

Jorge Burneo LONDON, ON

Richard Desbiens QUEBEC CITY, QC

David Fortin SHERBROOKE, QC

Mark Hamilton CALGARY, AB

Hans-Peter Hartung DUSSELDORF, GERMANY

Michael Hill CALGARY, AB

Alan C. Jackson WINNIPEG, MB

Daniel Keene OTTAWA, ON

James Perry TORONTO, ON

Oksana Suchowersky CALGARY, AB

Brian Toyota VANCOUVER, BC

Brian Weinshenker ROCHESTER, MN, USA

Samuel Wiebe CALGARY, AB

Elaine Wirrell ROCHESTER, MN, USA

\section{SECTION EDITORS/CONSEIL DE RÉDACTION}

Neuroimaging Highlight/Neuroimagerie David Pelz LONDON, ON

Neuropathological Conference/Conférence sur la neuropathologie

Robert Hammond LONDON, ON

Book Review/Critiques de livres

Reflections/Reflets

Andrew Kirk SASKATOON, SK

Critically Appraised Topic Summaries

(CATS)

Jorge Burneo LONDON, ON

Mary Jenkins LONDON, ON

\section{Editorial Review Board/Conseil de}

Revue d'éditorial

Donald Brunet KINGSTON, ON

Jodie Burton CALGARY, AB

Lionel Carmant MONTREAL, QC

Colin Chalk MONTREAL, QC

K. Ming Chan EDMONTON, AB

Robert Chen TORONTO, ON

Joseph Dooley HALIFAX, NS

Paolo Federico CALGARY, AB

Daryl Fourney SASKATOON, SK

Hannah Glass SAN FRANCISCO, CA, USA

Alan Goodridge ST. JOHN's, NL

Ian Grant HALIFAX, NS

Alan Guberman OTTAWA, ON

John Hurlbert CALGARY, AB

Manouchehr Javidan VANCOUVER, BC

Patrick McDonald WINNIPEG, MB

Martin McKeown vanCOUver, BC

Joseph Megyesi LONDON, ON

Vivek Mehta EDMONTON, AB

Steven Miller vanCoUver, BC

Neelan Pillay CALGARY, AB

Christopher Power EDMONTON, AB

Alex Rajput SASKATOON, SK

Jean Raymond MONTREAL, QC

Gary Redekop VANCOUVER, BC

Mark Sadler HALIFAX, NS

Harvey Sarnat CALGARY, AB

John Stewart VANCOUVER, BC

Jeanne Teitelbaum MONTREAL, QC

Eve Tsai OTTAWA, ON

Shannon Venance LONDON, ON

Matt Wheatley EDMONTON, AB

Jerome Yager EDMONTON, AB

Journal Staff - Calgary, $A B$

Dan Morin, Chief Executive Officer

Maggie McCallion, Designer/

Production Coordinator

Cindy Leschyshyn, Editorial Coordinator

Advertising representative/Représentant de publicité:

Brett Windle

Corporate Development Coordinator

Tel (403) 229-9575 Fax (403) 229-1661

E-mail: brett-windle@cnsfederation.org

Printer/Imprimeur:

Unicom Graphics, 4501 Manitoba Road SE

Calgary, Alberta T2G 4B9
The official journal of: / La Revue officielle de:

The Canadian Neurological Society La Société Canadienne de Neurologie The Canadian Neurosurgical Society La Société Canadienne de Neurochirurgie The Canadian Society of Clinical Neurophysiologists La Société Canadienne de Neurophysiologie Clinique

The Canadian Association of Child Neurology L'Association Canadienne de Neurologie Pédiatrique

The permanent secretariat for the four societies and the Canadian Neurological Sciences Federation is at:

Le secrétariat des quatre associations et du Fédération des sciences neurologiques du Canada est situe en permanence à:

7015 Macleod Trail SW, Suite 709

Calgary, Alberta, Canada T2H 2K6

CNSF (403) 229-9544 / CJNS (403) 229-9575 Fax (403) 229-1661

The Canadian Journal of Neurological Sciences is published bimonthly. The annual subscription rate for Individuals (print and online) are: C $\$ 170.00$ (Canada), C $\$ 200.00$ (US), C\$280.00 (International). Subscription rates for Institutions (print and online) are C $\$ 190.00$ (Canada), C $\$ 220.00$ (US), C $\$ 300.00$ (International). "Online Only"- Available only to International subscribers C $\$ 160.00$ (Individual), C $\$ 180.00$ (Institutional). See www.cjns.org for full details including taxes. Single copies C $\$ 30.00$ each plus C\$25.00 shipping and handling.E-mail: journal@cjns.org. COPYRIGHTC 2012 by THE CANADIAN JOURNAL OF NEUROLOGICAL SCIENCES INC. All rights reserved. No part of this journal may be reproduced in any form without the prior permission of The Canadian Journal of Neurological Sciences. Postage paid at Calgary, Alberta.

Le Journal canadien des sciences neurologiques est publié tous les deux mois. Voici les prix d'abonnement pour les personnes (imprimé et en ligne) : 170,00 \$ C (Canada), 200,00 \$ C (É.-U.), $280,00 \$ C$ (international). Voici les prix d'abonnement pour les institutions (imprimé et en ligne) : 190,00 \$ C (Canada), 220,00\$ C (É.-U.), 300,00 \$ C (international). «En ligne seulement » (offert seulement aux abonnés internationaux) : 160,00 \$ C (personnes), 180,00 \$C (institutions). Visiter www.cjns.org pour tous les détails incluant les taxes. Exemplaires uniques : $30,00 \$ C$ l'unité, plus $25,00 \$ \mathrm{C}$ en frais de port et de manutention. Courriel : journal@cjns.org. COPYRIGHT (C) 2012 du THE CANADIAN JOURNAL OF NEUROLOGICAL SCIENCES INC. Tous droits réservés. Aucune partie de ce journal ne peut être reproduite sous quelque forme que ce soit sans la permission préalable du Journal de la Fédération des sciences neurologiques du Canada. Frais de port payés à Calgary, en Alberta.

This journal is indexed by / Cette Journal est cité et indexé dans: Adis International, ArticleFirst, BIOBASE, BiolAb, BiolSci, BIOSIS Prev, Centre National de la Recherche Scientifique, CSA, CurAb, CurCont, De Gruyter Saur, E-psyche, EBSCO, Elsevier, EMBASE, FRANCIS, IBZ, Internationale Bibliographie der Rezensionen Geistes-und Sozialwissenschaftlicher Literatur, MEDLINE, MetaPress, National Library of Medicine, OCLC, PE\&ON, Personal Alert, PsycFIRST, PsycINFO, PubMed, Reac, RefZh, SCI, SCOPUS, Thomson Reuters, TOCprem, VINITI RAN, Web of Science.

ISSN 0317 - 1671 


\title{
Canadian Guidelines on Parkinson's Disease Introduction
}

\author{
David Grimes $^{1^{*}}$, Joyce Gordon ${ }^{2}$, Barbara Snelgrove ${ }^{2}$, Ivy Lim-Carter ${ }^{2}$, \\ Edward Fon ${ }^{3}$, Wayne Martin ${ }^{4}$, Marguerite Wieler ${ }^{4}$, Oksana Suchowersky ${ }^{5}$, \\ Alex Rajput ${ }^{6}$, Anne L. Lafontaine ${ }^{7}$, Jon Stoess ${ }^{8}$, Elena Moro ${ }^{9}$, Kerrie Schoffer ${ }^{10}$, \\ Janis Miyasaki ${ }^{9}$, Doug Hobson ${ }^{11}$, Minoo Mahmoudi ${ }^{12}$, Susan Fox ${ }^{9}$, \\ Ron Postuma ${ }^{7}$,Hrishikesh Kumar ${ }^{13}$, Mandar Jog ${ }^{13}$
}

Can J Neurol Sci. 2012;39: Suppl 4: S1-S30

The aim of the Canadian Guidelines on Parkinson's Disease is to enhance the care for all Canadians with Parkinson's Disease that:

- is based on the best published evidence

- involves expert consensus when there is a lack of evidence

- offers practical clinical advice

- takes into account patient choice and informed decision making

- is relevant to the Canadian Health Care System

The target users for the final document are health care professionals. However, they may also be used by stakeholders (policy makers, funding bodies) and people with Parkinson's disease and their families. These recommendations are intended to serve as a guide for health care providers, and clinical discretion should be used by all who are following the Canadian Parkinson's Guideline recommendations. The definitive judgment is made by the appropriate healthcare professional(s) based on all the data available for an individual person. It is recognized that resource problems may make it difficult to put into practice every recommendation in these guidelines. However, they are meant to improve the standard of care and access to care for individuals with Parkinson's in all regions of Canada.

A national meeting was held in January 2008 to bring together both clinicians and researchers to develop a comprehensive and unified Canadian network to collaborate, promote and advocate for excellence in clinical care and research in Parkinson disease. It was at this meeting that it became clear that most individuals in Canada are not seen at a specialized Parkinson's clinic. Guidelines are not meant to be a substitute for expert evaluation and management and that wherever possible, referral to a specialized, multidisciplinary clinic is still recommended. However, a tool was needed so that all health care providers who treat people with Parkinson's in Canada have a clear idea on how best to manage the disease. A second meeting was held in June
2008 to formally discuss if other guidelines and recommendations published on Parkinson's disease were applicable to the Canadian health care system. It was clear that Canadian guidelines were needed. While these could be based on other published recommendations, it was also clear that they need to be relevant from a Canadian perspective and that they need to be easily accessed by all health care providers.

The Canadian guidelines were developed with input from movement disorder specialists (with representatives from most of the major movement disorders centers in Canada), functional surgery specialists, family physicians, nurses, methodologists, physiotherapists, and the Parkinson Society Canada as well as individuals with Parkinson's. Conflicts of interest were recorded for all individuals that were part of the process and no participants or authors received any funding for their creation. The initial meeting expenses were in part supported with unrestricted grants from: Parkinson Society Canada, Parkinson Society of Southern Alberta, Hotchkiss Brain Institute University of Calgary, University of Ottawa, Novartis and Teva Canada Innovation. These guidelines have been endorsed by the Canadian Neurological Sciences Federation and Parkinson Society Canada.

These guidelines were completed in 2011 and will be reviewed in 2014. Comments are invited to assist the review process. Future work needed as part of these guidelines includes the creation of performance measures and methods to better assess outcomes in individuals with Parkinson's disease. All correspondence and comments regarding the recommendations in this Guideline should be sent to:

David Grimes, MD, FRCPC, Project Leader

1053 Carling Ave, C2 Rm 2194

Ottawa, ON, Canada

Phone: (613) 761-5353

Fax: (613) 761-5360

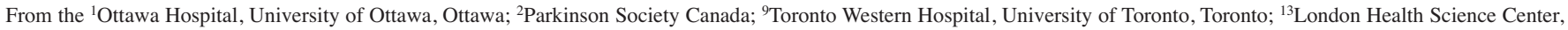
University of Western Ontario, London Health Science Center, London, Ontario; ${ }^{3}$ Department of Neurology, Montreal Neurological Institute; ${ }^{7}$ Montreal General Hospital, McGill University, Montreal, Quebec; ${ }^{4}$ Glenrose Rehabilitation Hospital, ${ }^{5}$ University of Alberta Hospital, University of Alberta, Edmonton, Alberta; ${ }^{8}$ Vancouver Hospital \& Health Science Centre, University of British Columbia, Purdy Pavilion, Vancouver, British Columbia; ${ }^{10}$ QEII Health Sciences Centre, Dalhousie University, QEII Health Science Centre Halifax, Nova Scotia; ${ }^{11}$ Movement Disorder Program, ${ }^{12}$ Department of Psychiatry, University of Manitoba, Winnipeg, Manitoba, Canada.

Received November 16, 2011. Final Revisions Submitted March 5, 2012

Correspondence to: D. A. Grimes, The Ottawa Hospital, Civic Campus, 1053 Carling Ave, Ottawa, K1Y 4E9, Canada. Email: dagrimes@ottawahospital.on.ca 


\section{Guideline Development Process}

The guidelines were developed using the ADAPTE (www.adapte.org) process and with the assistance of Dr. J Grimshaw (Director, Centre for Best Practices, Institute of Population Health, University of Ottawa). ADAPTE is a systematic methodology to aid in the adaptation of guidelines to help ensure the quality and validity of the adapted guideline, enhance the relevance of the adapted guideline to the context of use and to encourage confidence in and acceptance of the guidelines by targeted users.

A comprehensive search to identify previously published guidelines on Parkinson's disease was performed using the National Guideline Clearinghouse, National Library of Guidelines, CMA Infobase: Clinical Practice Guidelines, TRIP, Medline, Scirus, and Google. The search was limited to the years 2000 to September 2008 and had to be published in English or French. Articles related to Parkinson's clinical practice guidelines were obtained and their reference lists were reviewed to identify additional sources. Identified guidelines had to have more than one author and cited scientific evidence for the recommendations. This search identified eight publications that were relevant which were produced by three separate groups: The American Academy of Neurology; the European Federation of Neurological Societies; and the Movement Disorder Society-European Section and the National Institute for Health and Clinical Excellence from the United Kingdom (See Table 1). No additional guidelines were identified that met these criteria.

Prior to the national consensus conference that was held in January 2009, each clinical practice guideline was allocated to at least three expert panel members for appraisal of the guideline development process and quality of the guideline. This was done using the Appraisal of Guidelines for Research and Evaluation (AGREE) instrument (www.agreecollaboration.org). The AGREE instrument assessed the quality of guidelines across six domains including: (1) Scope and purpose; (2) Stakeholder involvement; (3) Rigour of development; (4) Clarity of presentation; (5) Applicability; and (6) Editorial independence. Each guideline was given a standardized score ranging from 1100 (100 representing a strong score) by the reviewing expert. The AGREE scores were then summarized (see supplemental Table 4).

The goal was not to create new recommendations, but to select from currently published, high quality guidelines whose recommendations were most clinically relevant for health care in Canada. Significant effort was made to try and maintain the phrasing of original recommendations but some have been modified slightly to achieve standardized terminology or to make the recommendation more specific. Care was taken to not change the context for which the original recommendation was written and no new Canada only recommendations were created. The source for all the original recommendations (NICE, AAN or EFNS) is referenced at the end of each Canadian recommendation. The systems for determining the level of evidence that were used across the guidelines differed slightly but the grade for the recommendation was maintained from the original source ie Grade A, B, C, D, U, or GPP (see supplemental appendix Tables 1-3 for detailed grade system for each guideline). For a simplified overview of the recommendations grades see Table 2. The most comprehensive guidelines were from the National Institute for Health and Clinical Excellence (NICE) in the UK and these served as the basis for the development of an outline and the original voting matrix used by the Canadian development group.

During the consensus meeting, separate working groups were created and were charged with reviewing all relevant material and recommendations on their topic (see Table 3 for guideline development participants). They then presented their

Table 1: Clinical Practice guidelines evaluated for the Canadian Guidelines

\begin{tabular}{l|l|l}
\hline Group & Guideline title & Year \\
\hline American Academy of Neurology & $\begin{array}{l}\text { Diagnosis and prognosis of new onset Parkinson disease (an } \\
\text { evidence-based review). }\end{array}$ & 2006 \\
\hline American Academy of Neurology & $\begin{array}{l}\text { Evaluation and treatment of depression, psychosis, and dementia in } \\
\text { Parkinson disease (an evidence-based review) }\end{array}$ & 2006 \\
\hline American Academy of Neurology & $\begin{array}{l}\text { Treatment of Parkinson disease with motor fluctuations and } \\
\text { dyskinesia (an evidence-based review): }\end{array}$ & 2006 \\
\hline American Academy of Neurology & $\begin{array}{l}\text { Neuroprotective strategies and alternative therapies for Parkinson } \\
\text { disease (an evidence-based review). }\end{array}$ & 2006 \\
\hline American Academy of Neurology & $\begin{array}{l}\text { Initiation of treatment for Parkinson's disease: an evidence-based } \\
\text { review. }\end{array}$ & 2002 (update 2005) \\
\hline $\begin{array}{l}\text { European Federation of Neurological } \\
\text { Societies and the Movement Disorder } \\
\text { Society-European Section }\end{array}$ & $\begin{array}{l}\text { Review of the therapeutic management of Parkinson's disease. Part I: } \\
\text { early (uncomplicated) Parkinson's disease }\end{array}$ & 2006 \\
\hline $\begin{array}{l}\text { European Federation of Neurological } \\
\text { Societies and the Movement Disorder } \\
\text { Society-European Section }\end{array}$ & $\begin{array}{l}\text { Review of the therapeutic management of Parkinson's disease. Part } \\
\text { II: late (complicated) Parkinson's disease. }\end{array}$ & 2006 \\
\hline $\begin{array}{l}\text { National Institute for Health and Clinical } \\
\text { Excellence - United Kingdom }\end{array}$ & $\begin{array}{l}\text { Parkinson's disease: diagnosis and management in primary and } \\
\text { secondary care }\end{array}$ \\
\hline
\end{tabular}


Table 2: Simplified Grading scheme from NICE, EFNS and AAN Guidelines

\begin{tabular}{l|l}
\hline $\begin{array}{l}\text { Recommendation } \\
\text { grade }\end{array}$ & Evidence \\
\hline A & $\begin{array}{l}\text { - Established as effective, ineffective, or harmful for } \\
\text { the given condition in the specified population. }\end{array}$ \\
\hline B & $\begin{array}{l}\text { - Probably effective, ineffective, or harmful for the } \\
\text { given condition in the specified population. }\end{array}$ \\
\hline C & $\begin{array}{l}\text { Possibly effective, ineffective, or harmful for the } \\
\text { given condition in the specified population. }\end{array}$ \\
\hline D & \begin{tabular}{l} 
Expert opinion, formal consensus. \\
\hline U
\end{tabular} \\
\hline GPP & $\begin{array}{l}\text { Data inadequate or conflicting given current } \\
\text { knowledge, treatment is unproven. }\end{array}$ \\
\hline
\end{tabular}

recommendations back to the entire group for further discussion and this served as the basis for the initial voting matrix for each recommendation. An open voting process and summary discussion method identified four main areas on which to base the guidelines: communication, diagnosis and progression, treatment of motor symptoms and non-motor features and their treatment. The voting matrix was then organized into these four main themes with subsections (See Table 4). Once the new matrix was constructed two phone conferences were held to ensure that the majority (more than 80 percent) agreed on each of the recommendation points. The recommendations and section summaries were then reviewed externally by two Parkinson's disease experts who had not participated in any previous step of the guideline development process (see Table 5 for authors and external reviewers).

The level of evidence and number of references varies considerably for the sections. The communications chapter is based solely on expert opinion and makes very broad statements but it is still important that these types of points are considered by all of those involved in the care of Parkinson's individuals. The studies used to generate the original source recommendations are not referenced separately but can be found from the guidelines that produced them. The authors for each section were instructed to limit references to only key information discussed if it was older than original source guideline. Some sections contain significant new information that was not available when the original recommendations were developed and therefore are typically the ones that have more discussion and references. This new information would not change the Canadian recommendation made but could have changed the grade level of the original source recommendation. The level of evidence quoted at the end of each recommendation however remains that of the source guideline to limit any confusion as to the grade recommendation source.

\section{Dissemination and Implementation}

During the development of these guidelines, consideration has been given to various methods of dissemination and the practical issues of implementation of each recommendation in a Canadian context. Consultation from other guideline groups in Canada has been elicited and presentation of draft forms of the guidelines has been done at neuroscience grand rounds within Canada. The Parkinson Society Canada will be assisting in the dissemination

Table 3: Canadian Guidelines Development Participants

\begin{tabular}{l|l|l}
\hline \multicolumn{1}{c|}{ Name } & \multicolumn{1}{|c}{ Job Description } & Affiliation \\
\hline Jennifer Conway & Nurse coordinator & Ottawa Hospital \\
\hline Dianne Cote & Nurse coordinator & Ottawa Hospital \\
\hline Jim Emmett & Family physician & Board Member, Parkinson Society Canada \\
\hline Edward Fon & Movement Disorder Neurologist & Montreal Neurological Institute \\
\hline Susan Fox & Movement Disorder Neurologist & Toronto Western Hospital \\
\hline Joyce Gordon & President and CEO & Parkinson Society Canada \\
\hline Doug Hobson & Movement Disorder Neurologist & Movement Disorder Clinic Deer Lodge Centre \\
\hline Karen Hunka & Nurse Coordinator & Foothills Hospital \\
\hline Mandar Jog & Movement Disorder Neurologist & London Health Science Center \\
\hline Anne Louise Lafontaine & Movement Disorder Neurologist & Montreal General Hospital \\
\hline Ivy Lim-Carter & $\begin{array}{l}\text { Director, National Research \& Clinical } \\
\text { Programs }\end{array}$ & Parkinson Society Canada \\
\hline Jon Stoessl & Movement Disorder Neurologist & Vancouver Hospital \& Health Science Centre \\
\hline Wayne Martin & Movement Disorder Neurologist & Glenrose Rehabilitation Hospital \\
\hline Janis Miyasaki & Movement Disorder Neurologist & Toronto Western Hospital \\
\hline Elena Moro & Functional Surgery Program Director, & Toronto Western Hospital \\
\hline Ron Postuma & Meurologist & Montreal General Hospital \\
\hline Emmanuelle Pourcher & Movement Disorder Neurologist & Clinique Sainte-Anne \\
\hline Alex Rajput & Movement Disorder Neurologist & Royal University Hospital \\
\hline Kerrie Schoffer & Movement Disorder Neurologist & Queen Elizabeth II Health Science \\
\hline Barbara Snelgrove & Director, Education \& Services & Parkinson Society Canada \\
\hline Oksana Suchowersky & Movement Disorder Neurologist & University of Alberta Hospital \\
\hline Marguerite Wieler & Physiotherapist/ Research Associate & Glenrose Rehabilitation Hospital \\
\hline & &
\end{tabular}


of the guidelines in print and electronic means to health care providers, individuals with Parkinson's and their families as well as posting the full guidelines on their website. They will be presented at national, provincial and regional meetings of healthcare professionals across disciplines. Feedback from these presentations will be encouraged to identify local and national barriers as well as ways to enhance their implementation. As part of the Parkinson Society Canada affiliation with the Neurological Health Charities Canada the guidelines will assist in advocacy efforts to federal and provincial governments to improve the care of individuals with Parkinson's as well as other brain disease. Responding to the paucity of information for neurological conditions, including Parkinson's, the Neurological Health Charities Coalition has partnered with the Public Health Agency of Canada, Health Canada, and the Canadian Institutes of Health Research to undertake the first-ever National Population Health Study of Neurological Conditions. This research initiative will gather data about the incidence, prevalence, risk factors, coexisting conditions, impact and services related to neurological conditions in Canada. It is expected that the study will be completed in 2013 and will help shape future updates to these guidelines.

A clear limitation to the implementation of these guidelines is a lack of adequate access to health care providers with expertise in dealing with individuals with Parkinson's. This includes not only specialty physicians but also nurses, speech, occupational and physical therapists with adequate training to deal with these very complex patients. Access to palliative care treatment is also lacking for Canadians with neurodegenerative disease and needs to be addressed at local and national levels of care delivery. Resource management, particularly in advanced stages, could be a potential ethical issue. Deep brain stimulation therapy is expensive and currently most centers do not have an indefinite budget to perform these procedures. Future treatments, like the
Table 4: Canadian Guidelines on Parkinson's Disease Overview

Section 1. Communication

Section 2. Diagnosis and Progression

Section 3. Treatment

A. Pharmacological therapy for motor symptoms in early PD

B. Pharmacological therapy for motor symptoms in later PD

C. Surgery

D. Other Treatment Options

Section 4. Non-motor Features and Their Treatment

A. Mental Health

B. Sleep disorders

C. Autonomic dysfunction

direct infusion of levodopa into the upper small intestine, may receive approval for use in Canada yet will cost tens of thousands of dollars per year. Should these be available options only for individuals who can pay for them privately? The cost of care for neurodegenerative diseases in general will increase as our population ages. Decisions about the limits that our public funded healthcare system can provide need to be addressed but are outside the scope of these guidelines.

\section{Abbreviations Used in Guideline Recommendations}

AAN - American Academy of Neurology

NICE - National Institute for Health and Clinical Excellence

EFNS - European Federation of Neurological Societies

$\mathrm{C} 1, \mathrm{C} 2$ etc - refer to the Canadian recommendation number for that point

Table 5: Authors and Reviewers

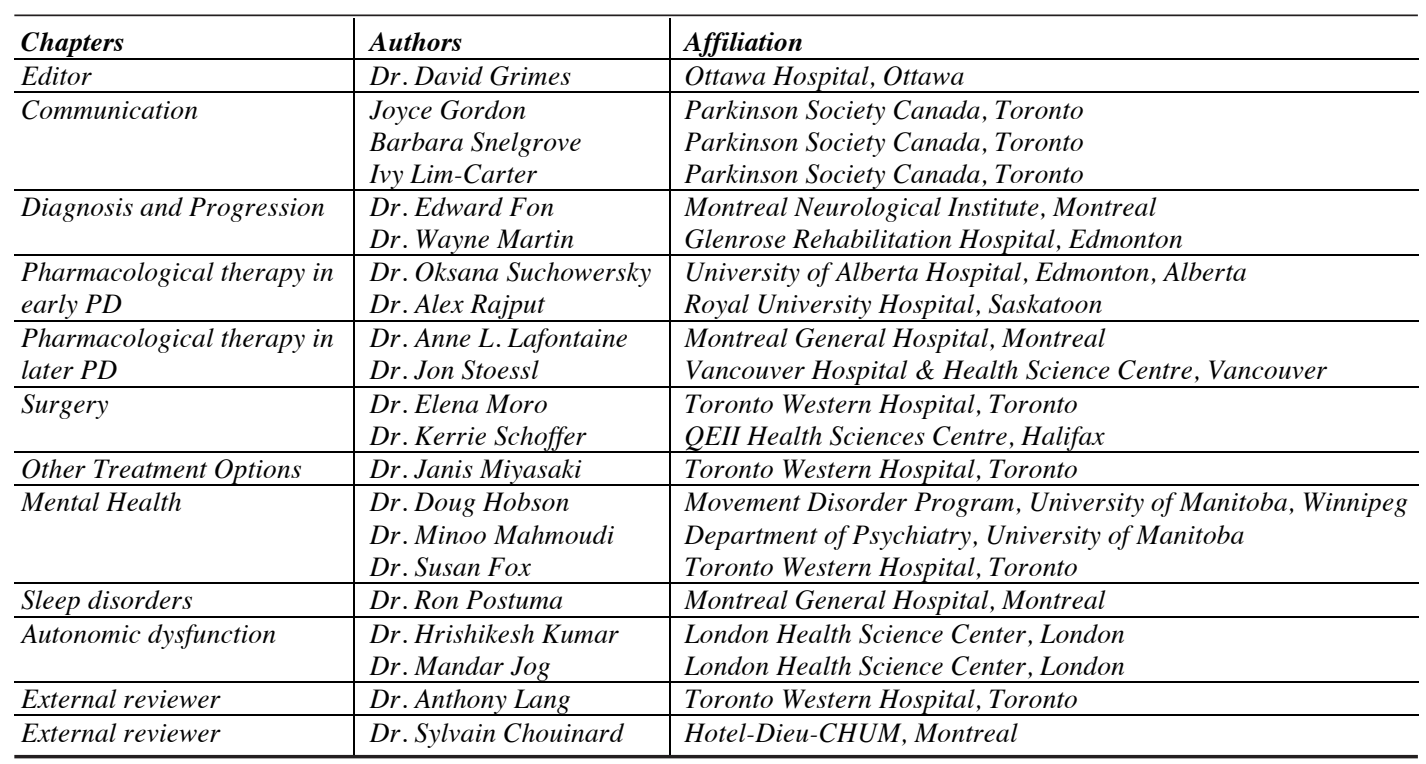




\section{Communication}

Good communication is at the heart of every interaction between people with Parkinson's, their caregivers and health professionals. Health care professionals committed to clear and compassionate communication can make a meaningful difference to their patients. When people with Parkinson's know what health care professionals recommend and why, they can anticipate what to expect; they are better prepared to navigate the system, ask the right questions, and make the best personal choices.

When a patient is newly diagnosed, health care professionals must have great sensitivity and understanding in describing the symptoms and progression of the disease. As it progresses, Parkinson's complicates every aspect of daily living. What were previously routine tasks now demand full attention and often result in frustration and anxiety. Parkinson's can compromise a person's ability to earn an income and can complicate relationships - with partners, family and friends. The progression of the disease leads to increased dependency which may lead to feelings of being a burden and increased strains on personal relationships. Stressors, such as threats to employment and social isolation are not uncommon. Beyond the need for medical care, people living with Parkinson's need understanding and support as they struggle to maintain independence and adapt to living with a chronic condition.

A person-centred approach to care and treatment should be cultivated for people living with Parkinson's. It relies upon open communication with health care professionals who are then able to provide quality care. People with Parkinson's should have the opportunity to make informed decisions based on full disclosure of all relevant information. Care decisions should be based upon best available evidence and provided by applicable professional standards.

Issues to consider when communicating with people with Parkinson's and their caregivers:

- Style, manner and frequency of communication that is compassionate and respectful

- Ease of access for those receiving information in a timely and appropriate manner throughout the progression of Parkinson's

- Honesty and sensitivity in tailoring information to meet changing medical needs

- Encouragement of self-management by people with Parkinson's to meet individual needs and preferences

- Inclusion of caregivers who are also impacted by Parkinson's and require information and support

Communication should be supported by the provision of evidence-based information offered in a form that is tailored to the needs of the individual. The treatment, care and information provided should be culturally appropriate and in a form that is accessible to people who have additional needs, such as people with physical, cognitive or sensory disabilities, and who do not speak or read English. Unless specifically excluded by the patient, caregivers and family members should have the opportunity to be involved in the discussion and decisions about the person's care and treatment.
C1 Communication with people with PD should be aimed towards empowering them to participate in the judgments and choices about their own care. NICE Level D

C2 Discussions should be aimed at achieving a balance between the provision of honest, realistic information about the condition and the promotion of a feeling of optimism. NICE Level D

C3 Because people with PD may develop impaired cognitive ability, a communication deficit and/or depression, they should be provided with: both oral and written communication throughout the course of the disease, which should be individually tailored and reinforced as necessary; and consistent communication from the professionals involved. NICE Level D (GPP)

The impact of Parkinson's is borne out in the many changes and accommodations that people living with Parkinson's and their caregivers have to make. Difficulty with writing, speaking, loss of independence, generally lead to social withdrawal and isolation as well as depression, frustration and anger. Access to services such as primary care, therapies for speech, exercise programs, and emotional support is critical to manage the disease and live with dignity.

An inter-disciplinary team approach in developing a care plan tailored to the unique needs of the individual is critical to maintain quality of life. Physicians and allied health professionals need to be knowledgeable about Parkinson's in order to provide specific services to the individual and these services should be coordinated from a central location.

Those in rural settings have more challenges accessing services and programs and must travel more distances to access these. Navigating the complex health and social service systems can be daunting. Health care professionals can help by understanding and being sensitive to the many challenges facing people living with Parkinson's and the potential barriers to accessing care and support. Parkinson's impacts both the person living with Parkinson's and the caregiver. It is important that both the person and the caregiver have access to the same information and services.

C4 Families and caregivers should be given information about the condition, their entitlements to care assessment and the support services available. NICE Level D (GPP)

C5 People with PD should have a comprehensive care plan agreed between the individual, their family and/or caregivers and all healthcare providers. NICE Level D (GPP)

C6 People with PD should be offered an accessible point of contact with specialist services. NICE Level D (GPP) 
There is little information with respect to palliative care in Parkinson's. Further, there are limited resources, including a lack of trained professionals, to adequately care for a person with Parkinson's over a long period of time, both in the community and in long-term care settings. In particular, physiotherapy, occupational therapy, speech therapy and dietician services are limited. In the advanced stage of Parkinson's, the emphasis of care should shift from an aggressive medical approach to a palliative care approach where the focus is providing comfort and support.

Traditionally, management of Parkinson's is focused on drug treatment and inter-disciplinary care for a long-term, slowly progressive disorder. Palliative care specialists have not routinely been involved. Due to the long duration of the disease and the difficulty in predicting the time of death, people with Parkinson's are frequently refused access to hospice and palliative care centres.
End-of-life choices, including advance care planning with an open and frank discussion with the patient and the person designated as decision-maker, should be initiated early in the disease process. The preparation of an advanced care directive should be discussed with the person with Parkinson's, and guidance and support should be provided to substitute decisionmakers who may have to make difficult decisions regarding lifesustaining treatment. If family and health care professionals have participated in a process of communication throughout the disease progression, the problems associated with interpretation and application of advanced directives are much less likely to occur.

C7 Palliative care requirements of people with PD should be considered throughout all phases of the disease. NICE Level D (GPP)

C8 People with PD and their caregivers should be given the opportunity to discuss end-of life issues with appropriate healthcare professionals. NICE Level D (GPP) 


\section{Diagnosis and Progression}

Parkinson's disease is characterized by a constellation of clinical manifestations, which include slowness of movement, rigidity, tremor and postural instability. Parkinson's disease is a complex disorder that can be difficult to diagnose clinically, especially in the early stages. Diagnosis based on etiology is impractical because no single cause of PD has been identified. For instance, both genetic and environmental factors involved in PD pathogenesis have been identified. However, established monogenic causes of PD account for only a minority of cases. Thus, currently the diagnosis of PD is based predominantly on the clinical features (Figure). Perhaps the most widely accepted clinical criteria for the diagnosis are those proposed by the UK Parkinson's Disease Society Brain Bank. ${ }^{1}$

C9 Parkinson's disease should be suspected in people presenting with tremor, stiffness, slowness, balance problems and/or gait disorders. NICE Level D (GPP)

There is no ideal way to define PD and distinguish it from other parkinsonian syndromes. Nevertheless, PD needs to be differentiated from other forms of parkinsonism, including multiple system atrophy (MSA), progressive supranuclear palsy (PSP) and corticobasal degeneration (CBD). Parkinson's disease should also be distinguished from secondary causes of parkinsonism such as drugs, neurotoxins, and structural brain lesions as well as other causes of tremor. Ideally, patients suspected of having PD or a related movement disorder, should be referred to a specialized movement disorders clinic or center for evaluation.

C10 Determining the presence of the following clinical features in early stages of disease should be considered to distinguish PD from other parkinsonian syndromes: 1) falls at presentation and early in the disease course; 2) poor response to levodopa; 3) symmetry at onset; 4) rapid progression (to Hoehn and Yahr stage 3 in three years); 5) lack of tremor; and 6) dysautonomia (urinary urgency/ incontinence and fecal incontinence, urinary retention requiring catheterization, persistent erectile failure or symptomatic orthostatic hypotension). AAN Level B

C11 People with suspected PD should be referred quickly* and untreated to a specialist with expertise in the differential diagnosis of this condition. NICE Level B

Parkinson's disease involves the degeneration of midbrain dopamine neurons, along with other catecholamine neurons, and the presence of Lewy bodies. However, Lewy bodies occur in individuals who do not have clinical features of PD and several forms of otherwise clinically typical PD do not have Lewy bodies. Moreover, relying on a neuropathology for diagnosis is not practical in life. About $20 \%$ of patients diagnosed with early stage PD have an alternative diagnosis at autopsy. ${ }^{2}$ Given the potential error in making a diagnosis of $\mathrm{PD}$, patients should be followed closely and the diagnosis reconsidered if atypical features emerge. In regions where appropriate facilities are available, consideration should be given to obtaining post mortem confirmation of diagnosis.

C12 Clinicians should be encouraged to discuss with patients the possibility of tissue donation to a brain bank for purposes of diagnostic confirmation and research. NICE Level D (GPP)

Development of reliable clinical or preclinical tests would greatly aid in the early identification of patients with PD or those at risk. Several drug challenges or diagnostic tests have been proposed to aid in the diagnosis of $\mathrm{PD}$ and/or in the differentiation between PD and other parkinsonian syndromes. However, to date, no single test has been shown to have sufficient sensitivity and specificity to reliably diagnose PD or distinguish PD from other forms of parkinsonism.

C13 There is insufficient evidence to determine whether levodopa challenge or olfaction testing have any advantage over the clinical diagnostic criteria of PD. AAN Level U

C14 The following may not be useful in differentiating PD from other parkinsonian syndromes: GH stimulation with clonidine, electrooculography, and SPECT scanning. AAN Level C

C15 There is insufficient evidence to support or refute the following as a means of distinguishing PD from other parkinsonian syndromes: urodynamics, autonomic testing, urethral or anal EMG, MRI, brain parenchyma sonography, and FDG PET. AAN Level U

Parkinson's disease is a heterogeneous disorder with clinical presentation varying substantially from patient to patient. A number of studies have examined the clinical PD subtype, the associated co-morbidities as well as response to treatment that were correlated with a more rapid progression of PD. This information may be helpful in guiding physician, patients and their families in the planning for long-term care.

C16 In patients with newly diagnosed PD, older age at onset and rigidity/hypokinesia as an initial symptom should be used to predict more rapid rate of motor progression. AAN Level B

C17 The presence of associated comorbidities (stroke, auditory deficits, and visual impairments), Postural Instability/Gait difficulty (PIGD), and male sex may be used to predict faster rate of motor progression. AAN Level C 
C18 Tremor as a presenting symptom may be used to predict a more benign course and longer therapeutic benefit to levodopa. AAN Level C

C19 Older age at onset and initial hypokinesia/rigidity should be used to predict earlier development of cognitive decline and dementia. AAN Level B

C20 Older age of onset, dementia, and decreased dopamine responsiveness may be used to predict earlier nursing home placement as well as decreased survival. AAN Level C

Parkinson's disease is a progressive disorder in which neuronal degeneration and clinical symptomatology tend to gradually worsen despite effective symptomatic treatment. While the most optimal treatment approach would alter the inexorable progression, an effective strategy has not yet been established. Clinical trials of putative neuroprotective compounds have been explored and, although some compounds show promise, results in general have been unrewarding, in part because of the challenges associated with establishing neuroprotection for compounds that may also have a symptomatic effect. ${ }^{3}$ Further trials are currently underway.
C21 Vitamin E should not be used as a neuroprotective therapy for people with PD. NICE Level A

C22 Co-enzyme Q10, dopamine agonists, monoamine oxidase B (MAO-B) inhibitors should not be used as a neuroprotective therapy for people with PD, except in the context of clinical trials. NICE Level B

C23 There is insufficient evidence to support or refute the use of amantadine or thalamotomy for neuroprotection. AAN Level U

C24 There is no long term evidence to recommend levodopa for neuroprotection. AAN Level U 


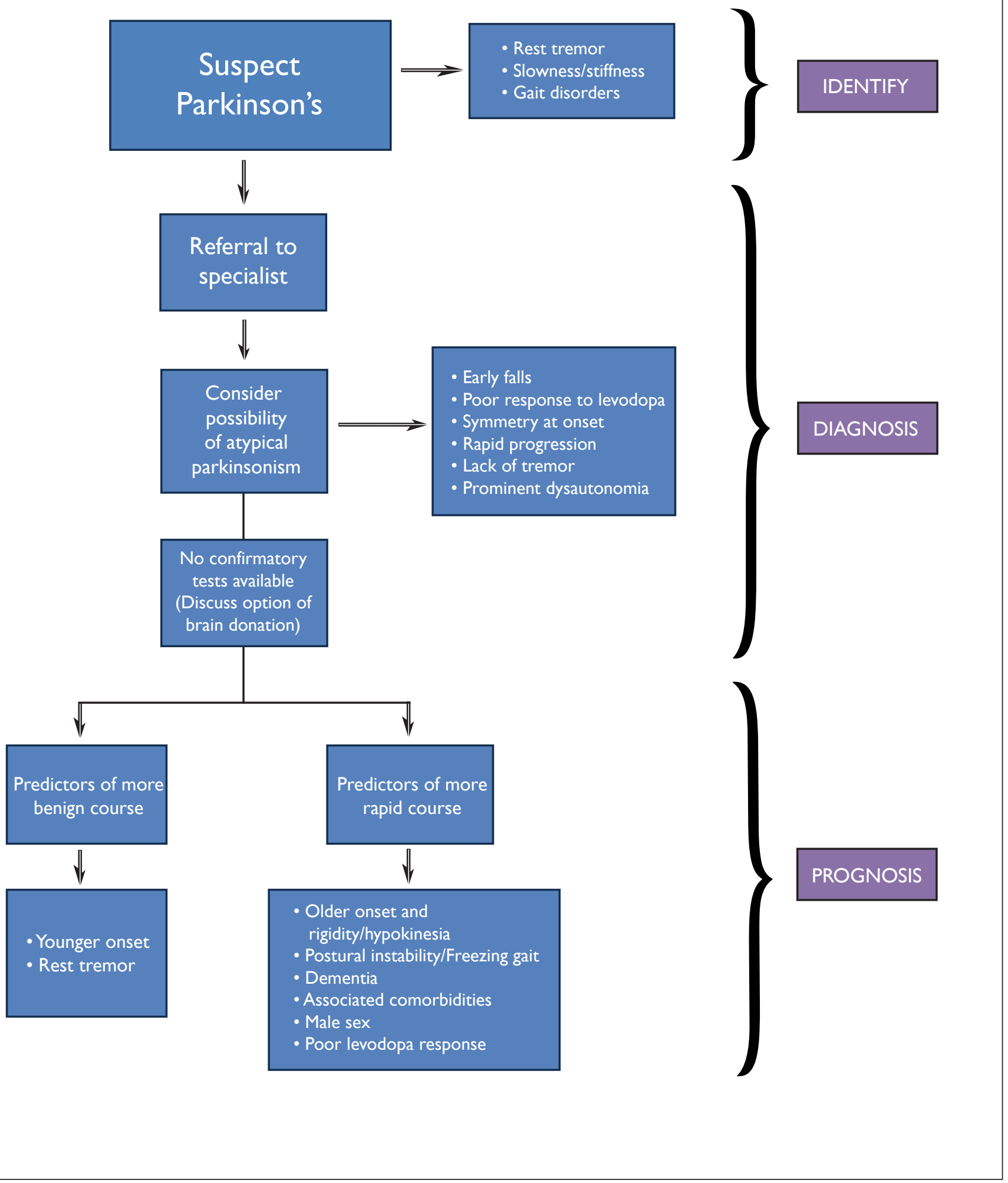

Figure: Summary of the identification, diagnosis and prognosis in Parkinson's disease

Suppl. 4 - S9 


\section{General Treatment Considerations}

There are a wide number of symptomatic treatments that are available for PD. These include medications, surgical procedures, physiotherapy, occupational therapy and other support services. All of these treatments can have a significant impact on improving an affected individual's quality of life and should be available. Despite the increase in non-pharmacological treatments, an individual with Parkinson's becomes more reliant on their medication to maintain their ability to function as the disease progresses. A balance between the side effects of the medication and the benefit often becomes more difficult with time. Medication schedules become more complex and the timing of when medications are given becomes crucial.

C25 Anti-parkinsonian medication should not be withdrawn abruptly or allowed to fail suddenly due to poor absorption (for example gastroenteritis, abdominal surgery) to avoid the potential for acute akinesia or neuroleptic malignant syndrome. NICE Level D (GPP)

C26 The practice of withdrawing patients from their antiparkinsonian drugs (so-called 'drug holidays') to reduce motor complications should not be undertaken because of the risk of neuroleptic malignant syndrome. NICE Level D (GPP)

C27 In view of the risks of sudden changes in anti-parkinsonian medication, people with PD who are admitted to hospital or care homes should have their medication: A) given at the appropriate times, which in some cases may mean allowing self-medication; B) adjusted by, or adjusted only after discussion with, a specialist in the management of PD. NICE Level D (GPP)

C28 Clinicians should be aware of dopamine dysregulation syndrome (impulse control disorders), an uncommon disorder in which dopaminergic medication misuse is associated with abnormal behaviours, including hypersexuality, pathological gambling and stereotypic motor acts. This syndrome may be difficult to manage. NICE Level D (GPP)

\section{A. Pharmacological Therapy for Motor Symptoms in Early PD}

Once the diagnosis of PD is made, the next decision is the type of treatment. The decision about initiation of pharmacologic therapy in PD patients should be tailored to the individual with the goal of reducing motor symptoms, and improving quality of life without causing side effects. There is no one medication which is recommended for treatment initiation, and factors that influence this decision include: symptom severity, whether the symptoms affect the dominant hand, embarrassment, ability to continue working and/or participate in activities such as hobbies, cost, and patient preference. If symptoms are very mild, the patient may choose not to begin therapy.

Some patients are resistant to starting dopaminergic medications due to concern about side effects or fear of limited duration of benefit. Currently, there is no evidence to suggest any of the medications, in particular levodopa, are toxic.

In fact, a good argument can be made that treatment should be initiated earlier rather than later for dopaminergic neuronal "sparing". 4

Medications effective for early symptomatic treatment that are currently available in Canada are listed in Table 6.

Levodopa remains the most effective medication for the treatment of motor symptoms. It is always given in combination with carbidopa ("Sinemet") or benserazide ("Prolopa") to prevent decarboxylation in the periphery. As it is associated with a higher risk for the development of motor complications (fluctuations

Table 6: Medications for the treatment of De Novo Patients

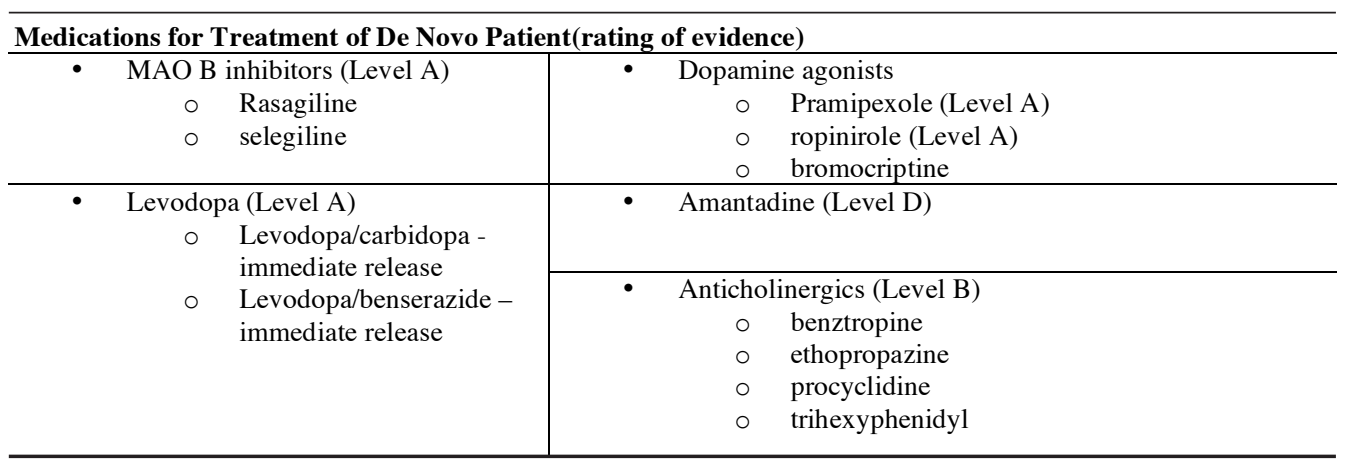


and dyskinesia), keeping the dose as low as possible to provide symptomatic benefit is generally recommended. A controlled release formulation of levodopa/carbidopa is available in Canada, but there is no evidence that it is superior to the regular formulation of levodopa/carbidopa in preventing motor fluctuations. A recent study has shown that using the combination with entacapone ("Stalevo") also does not delay the development of motor fluctuations. ${ }^{5}$

C29 It is not possible to identify a universal first-choice drug therapy for people with early PD. The choice of drug first prescribed should take into account:

- clinical and lifestyle characteristics

- patient preference, after the patient has been informed of the short- and long-term benefits and drawbacks of the drug classes. NICE Level D (GPP)

C30 Levodopa may be used as a symptomatic treatment for people with early PD. NICE Level A

C31 The dose of levodopa should be kept as low as possible to maintain good function in order to reduce the development of motor complications. NICE Level A

C32 Modified-release levodopa preparations should not be used to delay the onset of motor complications in people with early PD. NICE Level A

Dopamine agonists stimulate dopamine receptors directly, and unlike levodopa, do not need to be converted in the brain to be active. Dopamine agonists are the second most potent class of medication (after levodopa) for control of motor symptoms in PD with good evidence that they can be used in early PD with success. Dopamine agonists should be slowly titrated to a clinically effective dose. Adverse effects during upward titration may include nausea, lightheadedness, sleepiness and in some cases, hallucinations. Dopamine agonists have less likelihood of producing fluctuations in early disease, but are less effective, and are associated with a higher prevalence of side effects (hallucinations, leg edema, excessive daytime somnolence, impulse control disorders) and they are also more expensive than levodopa. ${ }^{6}$ In older patients, over the age of 70 , dopamine agonists should be used with caution, if not avoided. When using an ergot-derived agonist (bromocriptine is the only one currently available in Canada), baseline ESR, renal function, cardiac echocardiogram and chest X-ray are recommended prior to starting treatment and annually as long as the patient remains on the medication due to the risk of pleuropulmonary and cardiac valve fibrosis. As non-ergot derived agonists (pramipexole, ropinirole) do not carry this risk, or require this monitoring, they are preferred to an ergot-derived agonist. There is no good evidence that one dopamine agonist is superior to another regarding control of motor symptoms in PD. Thus, if one results in side effects, another could be substituted but the side effect profiles are similar.

C33 Dopamine agonists may be used as a symptomatic treatment for people with early PD. NICE Level A
C34 A dopamine agonist should be titrated to a clinically efficacious dose. If side effects prevent this, another agonist or a drug from another class should be used in its place. NICE Level D (GPP)

C35 If an ergot-derived dopamine agonist is used, the patient should have a minimum of renal function tests, erythrocyte sedimentation rate (ESR) and chest radiograph performed before starting treatment, and annually thereafter. NICE Level D (GPP)

C36 In view of the monitoring required with ergot-derived dopamine agonists, a non-ergot-derived agonist should be preferred in most cases. NICE Level D (GPP)

Monoamine-oxidase B inhibitors prevent the breakdown of dopamine in the brain. Two medications in this class are available in Canada: selegiline, and rasagiline. Each has been shown to have mild but definite symptomatic benefit as monotherapy in early PD. ${ }^{3}$

C37 MAO-B inhibitors may be used as a symptomatic treatment for people with early PD. NICE Level A

Amantadine is a medication with probably multiple, but poorly understood mechanisms of action. It may be used as monotherapy, but side effects such as livedo reticularis and leg edema need to be monitored. There is also a need to use this medication with caution in patients with renal dysfunction.

C38 Amantadine may be used as a treatment for people with early PD but should not be a drug of first choice. NICE Level D (GPP)

Anticholinergics, such as trihexyphenidyl and benztropine, are used primarily in young patients with early PD and prominent tremor. They are not recommended in the elderly, as they tend to cause confusion and memory difficulties.

C39 Anticholinergics may be used as a symptomatic treatment typically in young people with early PD and severe tremor, but should not be drugs of first choice due to limited efficacy and the propensity to cause neuropsychiatric side effects. NICE Level B

While the classical tremor seen in PD is a resting tremor, some patients have an associated postural tremor. Beta-blockers may be considered in this situation.

C40 Beta-adrenergic antagonists may be used in the symptomatic treatment of selected people with postural tremor in PD, but should not be drugs of first choice. NICE Level D (GPP)

\section{B. Pharmacological Therapy for Motor Symptoms in Later PD}

Levodopa is the most effective treatment for PD. In the early stages of disease, the clinical response to levodopa is prolonged; 
however, within a few years the duration of benefit from each dose may become progressively shorter. This phenomenon is referred to as "end of dose deterioration" or "wearing-off". Eventually patients may experience more unpredictable fluctuations including: on-off responses and freezing as well as involuntary movements broadly referred to as dyskinesias. These motor disabilities may have a significant impact on quality of life.

C41 It is not possible to identify a universal first-choice adjuvant drug therapy for people with later PD. The choice of adjuvant drug first prescribed should take into account:

- clinical and lifestyle characteristics

- patient preference, after the patient has been informed of the short- and long-term benefits and drawbacks of the drug classes. NICE Level D (GPP)

"End of dose deterioration" or "wearing-off" can be defined as the waning effect of levodopa within four hours of the last dose. Manipulation of the dose or frequency of levodopa can be a first strategy but eventually the emergence of dyskinesias will preclude this. Entacapone, a COMT inhibitor and rasagiline, a MAO-B inhibitor, have both been shown in clinical trials to reduce off time by approximately 1.5 waking hours per day. Entacapone is generally well tolerated but might exacerbate peak dose dyskinesias in which case, it is recommended to lower the dose of levodopa by $20 \%$. Tolcapone, the original COMT inhibitor in Canada has limited availability due to its associated hepatotoxicity. Rasagiline is also well tolerated but can lead to the emergence of dyskinesias. Although selegiline is pharmacologically similar to rasagiline, there are insufficient data from adequately controlled, randomized trials to recommend its use as an adjunct in the management of levodopa-related motor fluctuations.

C42 For patients with PD with motor fluctuations the available evidence suggests: Entacapone and rasagiline should be offered to reduce off time. AAN Level A

Dopamine agonists such as pramipexole, ropinirole and bromocriptine have been shown in clinical trials to reduce off time by approximately $15 \%$. In a single study, bromocriptine was found to have similar efficacy to pramipexole. Pramipexole and ropinirole have never been studied head to head. It is important to note that dopamine agonists can potentially cause significant side effects including drowsiness, sudden onset of sleep and impulse control disorders. It is recommended to carefully screen patients for pre-existing drowsiness and tendencies toward compulsive disorders such as gambling prior to prescribing dopamine agonists, as well as to monitor them for such potential problems throughout the course of treatment. Impulse control disorders are estimated to occur in more than $15 \%$ patients treated with dopamine agonists. ${ }^{6,7}$ Note that bromocriptine is another dopamine agonist that has been available in Canada for treating motor fluctuations for more than three decades. However, in addition to monitoring for all the usual side effects of dopaminergic therapy, physicians must warn patients about and remain observant for possible ergot toxicity, including erythromelalgia and fibrosis of serosal membranes. Cabergoline is another ergot derived dopamine agonist that is available, but not approved for the treatment of Parkinson's disease in Canada. Apomorphine can be administered by a variety of routes either intermittently, to treat sudden "off" periods unresponsive to adjustment of other medications, or by continuous infusion. However, its use in Canada is restricted to emergency release.

Transdermally administered dopamine agonists such as rotigotine ("the patch") are not currently available in Canada. The side effect profile and efficacy are in general similar to those of other dopamine agonists.

C43 Pramipexole and ropinirole should be considered to reduce off time. Pergolide is not available in Canada secondary to its association with valvular fibrosis. AAN Level B

Studies comparing immediate and modified-release preparations of levodopa have many methodological shortcomings but most have shown benefit in the management of "wearing off". It is important to remember that the overall amount of levodopa absorbed is roughly $25 \%$ less in the modified-release preparations of levodopa and this must be taken into account when switching between preparations. The side-effect profile is similar in both drugs. There is however a significant reduction in the number of doses per day with modified-release levodopa. In later PD, modified-release levodopa may be erratically absorbed, resulting in delayed-on or no-on responses. Modified release levodopa remains most useful in addressing overnight wearingoff. A variety of approaches have been used to provide levodopa by continuous enteral infusion, the most practical of which is in the form of a gel administered through a percutaneous enteral (PEG) tube. Although approved with conditions by Health Canada, this treatment is not available in Canada and the cost is likely to prohibit widespread use.

C44 Modified-release levodopa preparations may be used to reduce motor fluctuations in people with later PD but should not be drugs of first choice. NICE Level B

With advancing disease, dyskinesias become more frequent and more severe resulting in a considerable source of disability to many patients. The therapeutic window of levodopa becomes narrower so that small increases in dose to improve clinical effect result in dyskinesias. To date, only amantadine has been shown in clinical studies to improve dyskinesia without worsening parkinsonism. It is important to be aware of important cognitive side-effects that may arise as well as edema necessitating discontinuation of the drug.

C45 Amantadine may be considered for patients with PD with motor fluctuations in reducing dyskinesias. AAN Level C

\section{C. Treatment - Surgery}

The surgical treatment for PD is currently considered in advanced patients when the optimized medical treatment has failed in treating motor symptoms (such as motor fluctuations and/or dyskinesia). Surgical lesions of the basal ganglia such as thalamotomy for treating tremor and pallidotomy for levodopainduced dyskinesias were initially employed. However, lesions have been associated with a high risk of permanent side effects. 
Although pallidotomy and thalamotomy might still be performed in selected patients, deep brain stimulation (DBS) is currently the surgical treatment of choice in advanced PD patients. Compared to ablative surgery, DBS can be adjusted over time to address disease progression, has reversible effects, and can be used bilaterally to improve symptoms. The most used current targets for PD are: the thalamus (Vim nucleus), the subthalamic nucleus (STN), and the globus pallidus internus (GPi). The role of pedunculopontine nucleus stimulation is under investigation. Effectiveness of STN stimulation in improving levodoparesponsive signs and symptoms has been reported by many studies in the short-term and also in the long-term. ${ }^{8}$ The overall improvement of ADLs and motor UPDRS scores in the off medication/on stimulation condition has been reported to be on average $50 \%$ when compared to the off medications condition before surgery. ${ }^{8}$ Levodopa-induced dyskinesia has also been reduced by $69 \%$ on average after surgery. The most frequent adverse events (AEs) due to the surgical procedure include: infections $(6.1 \%)$, migration or misplacement of the leads $(5.1 \%)$, lead fractures $(5 \%)$, intracranial hemorrhage $(3 \%)$, and skin erosion (1.3\%). ${ }^{9}$ Several factors contribute to the DBS outcome, such as indications and patient selection, accuracy in surgical targeting, stimulation programming and medication management. The most reported complications possibly related to the stimulation (especially STN DBS) and persistent in the long-term follow-up include: eyelid opening apraxia (1.8-30\%), dysarthria/hypophonia (4-17\%), gait disturbances (14\%), postural instability $(12.5 \%)$ weight gain $(8.4 \%)$ and verbal fluency decline. ${ }^{10}$

In 2006 the AAN Subcommittee has found insufficient evidence to support or refute the efficacy of GPi or Vim DBS in improving off periods, dyskinesia or improving motor function. However, two large randomized multicenter studies comparing bilateral STN surgery to the best medical treatment have been recently published. ${ }^{11,12}$ Both these studies have shown that there was a significant improvement of motor function, dyskinesia and quality of life at six months in the DBS groups rather than in the medical groups, although the total number of adverse events was higher in the non-surgical groups. These results add more evidence that not only STN stimulation is superior to medical treatment in improving motor signs, but that DBS is also more effective in improving quality of life measures.

C46 DBS of the STN may be considered as a treatment option in PD patients to improve motor function and to reduce motor fluctuations, dyskinesia, and medication usage. Patients need to be counselled regarding the risks and benefits of this procedure. AAN Level C

Criteria of selection of patients' candidate for GPi DBS are similar to those for STN DBS. The issue as to whether STN is better than GPi stimulation in PD is still matter of discussion. In studies with short-term follow-up, GPi DBS patients have been reported to have less complications and similar or slightly less motor benefit. Results in the long-term have been more variable, and in one study a progressive loss of clinical benefit between the three and the five year follow-up was reported. ${ }^{13}$ These results emphasize the importance of long-term studies in order to clarify whether GPi can be an equal or a better target in selected PD patients.
C47 Bilateral GPi stimulation may be used in people with PD who:

- have motor complications that are refractory to best medical treatment

- are biologically fit with no clinically significant active comorbidity

- are levodopa responsive

- have no clinically significant active mental health problems, for example depression or dementia. NICE Level D

C48 With the current evidence it is not possible to decide if the STN or GPi is the preferred target for DBS for people with $\mathrm{PD}$, or whether one form of surgery is more effective or safer than the other. In considering the type of surgery, account should be taken of:

- clinical and lifestyle characteristics of the person with PD

- patient preference after the patient has been informed of the potential benefits and drawbacks of the different surgical procedures. NICE Level D

Recent studies have confirmed the long-term benefit of Vim DBS in PD patients with tremor. ${ }^{14}$ As expected, in these patients axial signs worsened over the years and there was progressive loss of benefit in activities of daily living (ADLs) compared to one year follow-up, likely related to the PD progression. Thus, there is general agreement that aged tremor-dominant PD patients with severe unilateral tremor can benefit more from Vim than STN DBS, due to the relatively less complicated thalamic procedure, simpler post-operative management, less stimulationrelated side effects and the usually relatively benign course of tremor-dominant PD.

C49 Thalamic DBS may be considered as an option in people with PD who predominantly have severe disabling tremor and where STN stimulation cannot be performed. NICE Level D

There are still no clear predictive factors of surgical benefit, except the pre-operative levodopa response. However, presurgical levodopa response was not predictive in a recent longterm follow-up study. ${ }^{8}$ A significant decline of the post-operative levodopa response over the years has been reported but this has been related to the progression of PD. ${ }^{8}$ As already noted above, there are no clear predictive factors of surgical outcome, except the pre-operative levodopa response for STN and GPi DBS.

C50 Preoperative response to levodopa should be considered as a factor predictive of outcome after DBS of the STN. AAN Level B

C51 There is insufficient evidence to make any recommendations about factors predictive of improvement after DBS of the GPi or VIM nucleus of the thalamus in PD patients. AAN Level U

Other unresolved issues concern the age and the PD duration at time of surgery. There is some evidence that PD patients $>70$ 
years may be at higher risk of post-operative cognitive decline, and less motor improvement compared to younger patients. Nevertheless, other studies do not report post-operative differences between younger and older STN DBS patients. ${ }^{15}$ One recent prospective randomized controlled study supports an early surgery in the course of PD, with the purpose to maintain good quality of life and high functional level. ${ }^{16}$

C52 Age and duration of PD may be considered as factors predictive of outcome after DBS of the STN. Younger patients with shorter disease durations may possibly have improvement greater than that of older patients with longer disease durations. AAN Level C

Treatments effective for motor complications in Parkinson's disease that are currently available in Canada are listed in Table 7.

Table 7: Treatment options for motor complications

\begin{tabular}{l|c}
\hline \multicolumn{1}{c}{ Treatment } & Level of Evidence \\
\hline \multicolumn{1}{c}{ Teduce Off Time } \\
\hline First Line & $\mathrm{A}$ \\
\hline Entacapone & $\mathrm{A}$ \\
\hline Rasagiline & $\mathrm{B}$ \\
\hline Pramipexole & $\mathrm{B}$ \\
\hline Ropinirole & \\
\hline Other Options & $\mathrm{B}$ \\
\hline Levodopa modified release & $\mathrm{C}$ \\
\hline DBS STN & $\mathrm{D}$ \\
\hline DBS GPi & \\
\hline Reduce Dyskinesia & $\mathrm{C}$ \\
\hline Amantadine & $\mathrm{C}$ \\
\hline DBS STN & $\mathrm{D}$ \\
\hline DBS GPi &
\end{tabular}

\section{D. Treatment - Other Treatment Options}

Previously, motor function received the primary attention of patients and physicians alike. This naturally led to concentration on pharmacologic therapies for PD. More recently, non-motor symptoms have become recognized as a major source of disability in PD and treatment focus has shifted to quality of life and maintaining it in advanced disease.

Thus, the focus on non-pharmacologic methods of treatment is emerging. Provision of education and valid information is essential to empower both patients and families in actively participating in disease management. Information sources include their physicians and nurse specialists with expertise in PD. In particular, nurses can provide psychological support, monitoring of medication adherence and in advanced stages, provide essential care including wound care monitoring when travel to clinics and hospitals becomes difficult. In this respect, Canada can learn much from the United Kingdom and other countries with active visiting nurse programs. United Kingdom nurses provide home visits and monitoring of apomorphine treatment, making this a viable alternative to surgery and delaying the need for alternative levels of care by maintaining mobility.

C53 People with PD should have regular access to the following:

- clinical monitoring and medication adjustment

- a continuing point of contact for support, including home visits, when appropriate

- a reliable source of information about clinical and social matters of concern to people with PD and their caregivers which may be provided by a Parkinson's disease nurse specialist. NICE Level C

Physical and exercise therapies may include multidisciplinary rehabilitation, active music therapy, treadmill training, balance training and "cued" exercise training. These all result in benefits, however continued therapy is required to sustain benefits. This is particularly important in PD since lack of motivation is a barrier to patient adherence in the absence of scheduled lessons or training. Caregivers may be unable to reinforce exercise programs at home, although home exercise programs have been shown to be effective. When enrolled in formal programs, patients showed improvement in ADL and motor scores, reduced bradykinesia (as measured by the Unified Parkinson Disease Rating Scale - UPDRS), improve ambulation speed (measured by the sit-stand-walk scores) and decreased falls. Given the large healthcare burden that falls represent and that those with PD have an increased risk of falls, use of exercise therapy in its various forms improves patients' safety, functional ability and therefore would presumably reduce overall healthcare expenditure.

C54 Physical and exercise therapies should be available for people with PD. Particular consideration should be given to:

- gait re-education, improvement of balance and flexibility

- enhancement of aerobic capacity

- improvement of movement initiation

- improvement of functional independence, including mobility and activities of daily living

- provision of advice regarding safety in the home environment. NICE Level B

Occupational therapy provides assessment of functional capacity and determines the best aids or strategies to improve functional capacity and therefore independence. Home safety is not easily assessed at an office visit. Home safety assessments and installation of appropriate aids such as shower seats, grab rails, poles at the bedside, removal of throw rugs, etc can improve the home environment for the patient and caregiver. Assessments can be provided by government agencies and aids are partially reimbursed through government assistive aid programs. 
C55 Occupational therapy should be available for people with PD. Particular consideration should be given to:

- maintenance of work and family roles, home care and leisure activities

- improvement and maintenance of transfers and mobility

- improvement of personal self-care activities, such as eating, drinking, washing and dressing

- environmental issues to improve safety and motor function

- cognitive assessment and appropriate intervention. NICE Level D (GPP)

Speech and language therapy is essential to Parkinson patient's quality of life. Hypophonia is a common problem resulting in social withdrawal and the misperception of cognitive decline for patients. Speech language therapy can improve vocal pitch and range leading to improved communication for patients. In advanced stages, assessment of swallowing safety is crucial. Speech language therapists in conjunction with clinical nutritionists make important contributions to the patient care team. Their involvement can result in identifying causes for weight loss, reduce the risk of aspiration and maintain weight.

C56 Speech and language therapy should be available for people with PD. Particular consideration should be given to:

- improvement of vocal loudness and pitch range, including speech therapy programs such as Lee Silverman Voice Treatment (LSVT). NICE Level B

- teaching strategies to optimize speech intelligibility NICE Level D (GPP)

- ensuring an effective means of communication is maintained throughout the course of the disease, including use of assistive technologies. NICE Level D (GPP)

- review and management to support safety and efficiency of swallowing and to minimize the risk of aspiration. NICE Level D (GPP)
Although many patients seek alternative therapies such as acupuncture, manual therapy or the Alexander technique, there is insufficient evidence to recommend these modalities. As patients' receptiveness and curiosity about alternative methods of treatment increases, the medical profession will need to respond with valid scientific data to provide guidance to patients. The placebo effect is well recognized particularly in PD and may in fact be mediated by dopamine. Therefore, any treatment involving PD must be subjected to rigorous scientific methods to establish efficacy and ensure that patients are receiving the best value for their time, effort and healthcare expenditure.

C57 There is insufficient evidence to support or refute the use of acupuncture, manual therapy, biofeedback or the Alexander technique in the treatment of PD. AAN Level U 


\section{Non-Motor Features of PD - Mental Health}

Neuropsychiatric symptoms are prevalent even prior to the motor symptoms of PD and become more prominent and increasingly challenging to treat with disease progression. They contribute to increasing disability and a negative impact on quality of life. The main symptoms addressed by this section include depression, dementia and psychosis. All these conditions are common in the general population without PD and for the most part are treated similarly in PD. Given the myriad of neurotransmitter changes present in PD it should not be assumed that standard pharmacologic treatment for these symptoms in patients without PD will be as effective or necessarily tolerated (eg dopamine antagonists for psychosis). Despite this, there is indeed a paucity of high level research trials to support the choice of symptomatic therapies for neuropsychiatric symptoms in PD. It should also be noted that there are many other neuropsychiatric manifestations in PD not addressed herein, including but not limited to anxiety, apathy and fatigue. The lack of sufficient research in the management of these additional problems prevents us from providing additional recommendations.

\section{Depression}

Depression is reported to occur in up to $50 \%$ of cases of PD and has been reported in up to $27 \%$ of early untreated cases. ${ }^{17}$ This neuropsychiatric problem has a major impact on both patient and caregiver quality of life. Due to the many overlapping features common to depression and PD, both prior to and while on treatment (loss of facial expression, hypophonic speech, slowed movement, reduced appetite and sleep disorders), depression in PD often goes on unrecognized. A high index of suspicion must be maintained for this non-motor symptom.

C58 Clinicians should have a low threshold for diagnosing depression in PD. NICE Level D (GPP)

C59 Clinicians should be aware that there are difficulties in diagnosing mild depression in people with PD because the clinical features of depression overlap with the motor features of PD. NICE Level D (GPP)

Symptomatic treatment of depression in PD has been poorly studied. There is not sufficient evidence in the literature to suggest treatment with levodopa will improve depression and only weak support for the efficacy using the dopamine agonist pramipexole. There have been antidotal reports of the MAO-B medication selegiline helping depression but this has yet to be confirmed in adequate studies. In general when symptoms of depression are confined to 'off time' they may respond well to any treatment that will reduce fluctuations and improve 'on time'.

Amitriptyline is to be considered in the treatment of depression in PD without dementia based on the AAN guidelines published in 2006. This could be a benefit seen with tricyclics in general as subsequent to this, a modest trial comparing nortriptyline with paroxetine CR in treatment of depression in PD concluded nortriptyline was efficacious and paroxetine $\mathrm{CR}$ was not. ${ }^{18}$ An additional single blind study comparing low dose amitriptyline $(25 \mathrm{mgs})$ to sertraline $(50 \mathrm{mgs})$ confirmed equal efficacy for depression but sertraline was more efficacious in improving quality of life. ${ }^{19}$ A small controlled trial of desipramine vs citalopram recently concluded they were similarly efficacious in PD depression vs placebo and both were more effective than placebo. ${ }^{20}$ The evidence for SSRIs as antidepressants in PD is thus still unclear. A recent RCT demonstrated no significant benefit of atomoxetine vs placebo ${ }^{21}$. ECT remains a potentially lifesaving treatment in major depression and has been used successfully in PD but sufficient trials in PD depression do not exist. More recently, the dopamine agonist, pramipexole (up to $1 \mathrm{mg}$ TID) significantly improved depression compared to placebo, an effect unrelated to motor improvement ${ }^{22}$. However, further studies are needed to confirm this class effect in depression in PD.

C60 The management of depression in people with PD should be tailored to the individual, in particular, to their co-existing therapy. NICE Level D (GPP)

C61 Amitriptyline may be considered in the treatment of depression associated with PD. AAN Level C

\section{Psychotic Symptoms}

Psychotic features occur in up to $50 \%$ of cases with Parkinson's disease and once evident, typically persist as a problem through the course of their disease. There is a typical progression from illusions of presence, through pseudo hallucinations (preservation of awareness of false nature of the phenomenon) to true hallucinations. Visual hallucinations are the most common although auditory hallucinations also occur. Paranoia is a common accompaniment.

Not all hallucinations require treatment. If they are sufficiently problematic to the patient or caregiver then alteration in treatment is needed. It is important to ensure the patient is in a safe, quiet, well lit, calming environment and that any precipitating medical problems are ruled out. Eliminating all nonessential central nervous system active medications is important and is often overlooked. If these steps do not control the hallucinations, then reducing or stopping parkinsonian medications that have a greater potential for worsening psychosis relative to the parkinsonian benefit may be needed. The risk of rapid discontinuation of dopaminergic medications worsening psychosis or causing potential neuroleptic malignant syndrome needs to be kept in mind and monitored for. 
If following the above suggestions is inadequate then the addition of an antipsychotic may be necessary. In choosing these, typical antipsychotic medications (phenothiazines, butyrophenones) should be avoided due to their potential for exacerbating PD motor symptoms. There is less agreement in the literature in regard to the use of atypical antipsychotics although olanzapine shouldn't be considered. Risperidone has not been favored unless quetiapine and clozapine have failed but robust enough information to conclude it is safe or not, is still lacking. ${ }^{23}$ Quetiapine may have a lower potential for causing worsening of parkinsonism and was considered as a safe treatment option but this conclusion is now questionable after two subsequent randomized trials failed to confirm this efficacy. ${ }^{24,25}$

Clozapine use is the most supported medication option in the treatment of psychosis in PD but is not easy to use. A small comparative trial of clozapine and quetiapine in PD psychosis demonstrated equal efficacy of quetiapine which would suggest it may still be considered first to avoid the more intensive monitoring required with clozapine. ${ }^{26}$ The quetiapine dose used was up to $150 \mathrm{mg} /$ day in this study.

An additional potential pharmacologic intervention that has been suggested for psychosis are the cholinesterase inhibitors rivastigmine and donepezil, however their use to date is not supported by any high level studies. There is concern that motor worsening, particularly tremor can occur with this class of medication. As a result any recommendations still await large, placebo-controlled investigation. ECT remains a potentially lifesaving treatment in psychosis and has been used successfully in PD but sufficient trials in PD psychosis do not exist.

C62 All people with PD and psychosis should receive a general medical evaluation and treatment for any precipitating condition. NICE Level D (GPP)

C63 Reduce polypharmacy. Reduce/stop anticholinergic antidepressants, reduce/stop anxiolytics/sedatives. EFNS (GPP)

C64 Consideration should be given to gradually withdrawing antiparkinsonian medication that might have triggered psychosis in people with PD. NICE Level D (GPP)

C65 Reduce antiparkinsonian drugs. Stop anticholinergics, stop amantadine, reduce/stop dopamine agonists, reduce/stop MAO-B and COMT inhibitors, lastly, reduce levodopa. Stopping antiparkinsonian drugs can be at the cost of worsening motor symptoms. EFNS (GPP)

C66 Mild psychotic symptoms in people with PD may not need to be actively treated if they are well tolerated by the patient and caregiver. NICE Level D (GPP)

C67 Typical antipsychotic drugs (such as phenothiazines and butyrophenones) should not be used in people with PD because they exacerbate the motor features of the condition. NICE Level D (GPP)

C68 For patients with PD and psychosis, olanzapine should not be routinely considered. AAN Level B
C69 Clozapine may be used in the treatment of psychotic symptoms in PD, but registration with a mandatory monitoring scheme is required. It is recognized that few specialists caring for people with PD have experience with clozapine. NICE Level B

C70 For patients with PD and psychosis, quetiapine may be considered. AAN Level C

\section{Dementia}

Dementia in PD is common, especially in those with an older age of onset and its frequency increases with disease duration. ${ }^{27}$ As patients with PD live longer this problem will become an increasingly difficult management problem.

As with psychosis, after ruling out other potential medical disorders contributing to dementia (thyroid dysfunction, B12 deficiency etc), it is generally recommended that a simplification of medications be undertaken to minimize potential untoward CNS effects that accentuate the cognitive dysfunction. There is evidence in favor of discontinuing anticholinergics, amantadine, tricyclics, tolterodine, oxybutinin and benzodiazepines.

There are several treatment options for symptoms of PD dementia with modest potential benefit. There has been enough research on the use of cholinesterase inhibitors donepezil and rivastigmine to suggest they should be considered for use. There is less evidence to support the use of memantine or galantamine in PD dementia. ${ }^{28-30}$

C71 Discontinue potential aggravators;

- Anticholinergics. EFNS Level B

- Amantadine, tricyclic antidepressants, benzodiazepines, tolterodine and oxybutynin. EFNS Level C

C72 Donepezil should be considered for the treatment of dementia in PD. AAN Level B

C73 Rivastigmine should be considered for the treatment of dementia in PD or Dementia with Lewy Bodies. AAN Level B

\section{B. Sleep Disorders}

A variety of sleep disorders affect patients with PD. The major sleep disorders in Parkinson's disease include insomnia, excessive daytime somnolence, REM sleep behavior disorder, and restless legs syndrome.

Insomnia - Experienced by up to $60 \%$ of patients with PD, insomnia is usually characterized by difficulty staying asleep (i.e. sleep maintenance insomnia), although sleep onset insomnia may also occur. ${ }^{31}$ The etiology of insomnia is multifactorial and can include motor symptoms of PD (e.g. pain, tremor), medication side effects, restless legs syndrome, depression, and nocturia. This necessitates a thorough history, to detect reversible causes. Insomnia may also be a primary feature of the disorder, as sleep regulating centers degenerate in $\mathrm{PD}$. 
C74 A full sleep history should be taken from people with PD who report sleep disturbance. NICE Level D (GPP)

Randomized controlled trial evidence for insomnia treatment is relatively limited. In general, non-pharmacologic treatment for insomnia consists of sleep hygiene measures and cognitive behavioral therapy. These have consistently been demonstrated as effective in primary (psychophysical insomnia), and also some forms of secondary insomnia (although they may be less effective than for primary insomnia). ${ }^{31,32}$ There have been no trials demonstrating utility of sleep hygiene techniques in PD, but many clinicians recommend them because of their proven utility in other forms of insomnia.

C75 Good sleep hygiene should be advised in people with PD with any sleep disturbance and includes:

- avoidance of stimulants (for example, coffee, tea, caffeine) in the evening

- establishment of a regular pattern of sleep

- comfortable bedding and temperature

- provision of assistive devices, such as a bed lever or rails to aid with moving and turning, allowing the person to get more comfortable

- restriction of daytime siestas

- advice about taking regular and appropriate exercise to induce better sleep

- a review of all medication and avoidance of any drugs that may affect sleep or alertness, or may interact with other medication (for example, selegiline, antihistamines, $\mathrm{H} 2$ antagonists, antipsychotics and sedatives). NICE Level D (GPP)

In terms of pharmacologic therapy, there have been two recent trials of melatonin for insomnia in PD - both showed modest benefit (e.g. improvement of sleep time by ten minutes in one study) - there was improvement of subjective measures, but conflicting improvement of objective measures. ${ }^{33,34}$ The effect of levodopa on sleep quality is also unclear - one randomized trial demonstrated benefit on self-reported sleep quality, whereas a second found mild improvement in self-reported sleep duration and nocturnal akinesia but no effect on overall sleep quality. ${ }^{35,36}$ A recent randomized trial suggested that eszopiclone may be partially useful for insomnia in PD. In this trial, 2-3 mg eszopiclone did not change total sleep time, fatigue, quality of life, daytime alertness, or caregiver stress, but did improve subjective quality of sleep, number of awakenings, and clinical global impression ${ }^{37}$. Other treatments of insomnia have not been tested in a randomized controlled trial. A recently-published guideline from the American Academy of Neurology states: ${ }^{38}$

"Levodopa/carbidopa improved sleep-associated motor symptoms that may contribute to insomnia, but data demonstrating an improvement in objective sleep parameters or sleep satisfaction are insufficient. Melatonin is established as effective in improving patients' perception of sleep quality (2 Class I studies) but data are conflicting regarding objective improvement in sleep quality as measured by PSG."

REM Sleep Behaviour Disorder (RBD) is characterized by the loss of normal muscle tone during REM sleep and is experienced by approximately $35 \%$ of patients with PD..$^{39.40}$ Patients act out their dream content and may punch, kick, talk, shout and fall out of bed. Injury is quite common. RBD can predate the diagnosis of PD and over $40 \%$ of patients with idiopathic RBD will be diagnosed with a neurodegenerative disease such as PD after ten years. The exact pathological mechanism is unclear but animal models implicate deficits in brainstem (especially pontine) nuclei controlling REM sleep. Observational studies have suggested that clonazepam (0.25-1mg hs) or melatonin (3-12mg hs) can suppress RBD. ${ }^{40}$ In a recent practice guide by the American Academy of Sleep Medicine, clonazepam and melatonin are recommended for the treatment of RBD. ${ }^{41}$ However, no randomized controlled trials have been performed of any agent for RBD.

Restless legs syndrome (RLS) is characterized by a sensation of urge to move the legs, which is worse at night, exacerbated by rest, and relieved by activity. RLS can be treated with dopaminergic agents, opioids, or gabapentin. ${ }^{42}$ It is unclear to what degree RLS frequency is increased in PD, and symptoms of RLS can overlap with other symptoms of parkinsonism. RLS can be exacerbated by iron deficiency, so clinicians should usually check ferritin levels in patients with RLS.

C76 Care should be taken to identify and manage restless legs syndrome (RLS) and rapid eye movement (REM) sleep behaviour disorder in people with PD and sleep disturbance. NICE Level D (GPP)

Excessive daytime sleepiness (EDS) is common in PD, affecting up to $40 \%$ of patients. ${ }^{43}$ In mild cases, patients fall asleep when inactive but when severe, patients fall asleep even in stimulating conditions such as eating, walking, or working. Sudden sleep attacks while driving have been reported. The etiology of EDS is multifactorial. Insomnia and fragmented sleep due to restless legs syndrome can cause somnolence during the day, although on average, patients with EDS sleep more deeply than those without. Medications, particularly dopaminergic medications, commonly exacerbate somnolence. Sleep apnea can present with somnolence, although it is not clear that mild apnea is an important cause of somnolence in PD. ${ }^{44}$ The primary reason for EDS, however, is degeneration of central sleep regulation centres in the brainstem - that is, EDS is a primary feature of PD.

There have been three trials of modafinil a psycho-stimulant with an unknown mechanism. Two clinical trials found an improvement in EDS in $\mathrm{PD}^{45,46}$; however, a third showed no effect. ${ }^{47}$ The amplitude of effect of modafinil is modest, with Epworth Sleepiness Scale improvement ranging from 1.2 to 4.4 relative to placebo. ${ }^{45,47}$ No other randomized trials of agents for EDS have been performed. Modafinil is not covered by many provincial drug plans, which may limit its use. Physicians are advised to be aware of their provincial legislation regarding driving in persons who are experiencing sleep attacks - in many provinces, inability to safely drive is legally reportable to licensing agencies.

C77 People with PD who have sudden onset of sleep should be advised not to drive and to consider any occupational hazards. Attempts should be made to adjust their medication to reduce its occurrence. NICE Level D (GPP) 
C78 Modafinil may be considered for daytime hypersomnolence in people with PD. NICE Level D(GPP)

\section{C. Autonomic dysfunction. Treatment - General considerations}

Autonomic dysfunction is extremely common in PD and encompasses cardiovascular, gastrointestinal, urogenital and thermoregulatory disorders. Estimated prevalence of these symptoms varies between $14 \%$ and $80 \%$ across PD patients depending upon the methodology of the studies. ${ }^{48}$ Autonomic dysfunctions specially orthostatic hypotension, nocturia and constipation have significant impact on quality of life. ${ }^{49}$ Despite the frequency and breadth of the symptoms evidence regarding specifics of management is poor. Due to the lack of randomized controlled trials across these symptoms, many of the recommendations are based on data from treating these symptoms in non-parkinsonian individuals.

Weight loss is common in PD and may occur in up to $70 \%$ of patients. ${ }^{50}$ There are many possible causes: dysphagia, dysmotility with delayed gastric emptying, constipation, dyskinesia and metabolic calorie loss, reduced appetite from loss of sense of smell, depression, cognitive impairment, immobility, non-appealing food textures (pureed or minced) and incorrect diet, reduced access to food and possibly side effects of medications such as nausea. ${ }^{50,51}$ Parkinson's disease being a chronic disease state may also be a contributor. No specific recommendations are available for weight loss but some of the above mentioned causes can be treated. A dietary consultation and dysphagia assessment may be useful. Antidepressants that improve mood may also be effective in causing weight gain. Nutritional supplementation with high calorie drinks (e.g. Ensure, Resource, Boost) may be suggested however, high protein drinks need to be kept away from levodopa. Whey protein drinks are also a source of nutrition.

Sialorrhea can be a functionally and cosmetically disabling symptom in PD. No specific recommendations had been suggested in the original reviewed guidelines however new evidence does exist for various treatment options. The American Academy of Neurology recently reviewed the use of botulinum neurotoxin in the treatment of autonomic disorders and concluded that it should be considered as an option for individuals with Parkinson's disease who are having difficulty with drooling. ${ }^{52}$ Atropine drops, ipratropium bromide spray and gum chewing have been evaluated in a more controlled and blinded fashion. ${ }^{53-}$ ${ }^{55}$ While the latter two are cheap and affordable, botulinum toxin A is not covered by most provincial formularies for sialorrhea injection and would be considered an off label use. A complete dysphagia assessment via videofluoroscopy and referral to speech language pathology should be considered if an individual presents with prominent drooling because of the significant correlation between drooling and oropharyngeal dysphagia. It is also important to ensure adequate oral hygiene practices are in place.

Swallowing difficulties can become an increasing concern as the disease progresses. Oropharyngeal dysphagia in the early stages may not require any specific investigations if it occurs infrequently (less than one time per week) and is limited to liquids. Treatment at this stage would include encouraging smaller boluses, having an increased attention to eating and reducing distractions at meals. Providing education on monitoring for signs/symptoms of dysphagia is important. Adequate oral hygiene practices need to be in place to reduce the risk of aspiration and the development of pneumonia.

As the dysphagia progresses (choking greater than one time per week) a referral for a formal assessment with speech language pathology should be considered. A formal videofluoroscopic evaluation may be necessary if dysphagia screening is failed or the individual is symptomatic with respiratory infection or weight loss. It is important that ongoing monitoring of respiratory and nutritional status occurs and referral to a registered dietician for nutritional support may be considered. Food consistency changes such as soften diet texture to minced diet, eliminate mixed consistencies (foods with both liquid and solid components e.g. cold cereal with milk), reduce bolus volume, and modify fluids to honey or nectar consistency are often needed. Safety of diet texture modifications and use of postures/maneuvers should be verified under videofluoroscopic evaluation. With severe dysphagia close monitoring with the involvement of speech language pathology and dietician are essential. Engaging patients and their families in quality of life discussions around enteral feeding options or enteral delivery of medication as required may be necessary. With worsening dysphagia severity, suctioning equipment for secretion management may be required.

For individuals with symptoms more of esophageal dysphagia screening for gastroesophageal reflux disease and other upper GI tract dysfunction is important. If individuals are more symptomatic consideration of a referral for upper GI/gastric motility studies or to a gastroenterologist is warranted. Reflux treatments or prokinetic (domperidone) medications for symptom management are helpful but it is important to remember to avoid the use of metoclopramide. Because oropharyngeal and esophageal dysphagia co-occur often in PD, be aware that patients demonstrating symptoms of both disorders require referral to speech language pathology and in addition to this may require a referral to gastroenterology.

Thermodysregulation and feeling hot and cold are frequent in PD. Excessive sweating (hyperhidrosis) may accompany these symptoms and can be unpredictable. Often, drenching sweats may occur at night. The pattern of hyperhidrosis in PD varies; it can be associated with motor "off" time, or with peak-dose dyskinesias. Treatment options for hyperhidrosis are limited ${ }^{9}$ but in patients with motor fluctuations it may improve with adjustment of PD medications. Medical conditions such as hyperthyroidism need to be excluded. Oral medications such as low doses of clonidine, tricyclics with anticholinergic side effects and beta blockers may be tried. ${ }^{38,56}$ Caution with respect to hypotension is warranted.

C79 People with PD should be treated appropriately for the following autonomic disturbances: NICE Level D (GPP)

- urinary dysfunction

- weight loss

- dysphagia

- constipation

- erectile dysfunction

- orthostatic hypotension

- excessive sweating

- sialorrhoea 


\section{Urinary Dysfunction}

The most common forms of urinary dysfunction are urgency, frequency and nocturia. In men, prostatic hypertrophy must be ruled out. A urological assessment is always warranted if pathology different than PD is suspected. Urologists are generally easily accessible in most centres. Simple treatment measures include regular visits to the bathroom at intervals such that urgency can be avoided. The consumption of water or caffeinated drinks after dinner is to be restricted to avoid nocturia. Easy access to the bathroom and if required a bedside urinal may be necessary to avoid incontinence. Finally a condom catheter may be needed in some cases. Application of the condom catheter can be taught to most caregivers by community care access services. Specific recommendations for treatment of these symptoms include anticholinergics. Oxybutynin and tolterodine are regularly used. ${ }^{57,58}$ However, in most cases the provincial health plan will not cover tolterodine if oxybutynin has not been tried first. Patients should be closely monitored for anticholinergic related side effects such as confusion, hallucinations, dry mouth and urinary retention. Newer anticholinergics such as solifenacin (Vesicare) may also be used. ${ }^{57-59}$

C80 General measures for treating urinary urgency and incontinence include avoiding coffee before bedtime, limit water ingestion before bedtime, etc.

- Add peripherally acting anticholinergic drugs. EFNS (GPP)

\section{Constipation}

Constipation can predate the onset of PD symptoms by decades. ${ }^{60}$ However, dysmotility in PD is not only a function of lower gastrointestinal (GI) dysfunction but also due to slowing of transit time through the entire GI tract. Evacuating stool is also an issue in many patients if the stool is hard. Again, good quality data is lacking for most suggested therapies for treating constipation in PD. This point is highlighted by the recent American Academy of Neurology practice parameters on the treatment of nonmotor symptoms that identified only macrogol as a treatment option (Level C). ${ }^{38}$ Simple measures such as ensuring a higher content of fibre including consumption of fruits and vegetables are crucial along with a high consumption of water. Simply consuming fibre without drinking water is not useful but care must be taken to not aggravate urinary symptoms. A wide variety of laxatives are available and include:

a) Bulk-forming laxatives draw water into the stool to create large soft stools. The larger stools help trigger the bowel to contract and move the stools out. Metamucil, psyllium are examples and these laxatives are generally gentle.

b) Lubricant laxatives coat the stool surface allowing the stools to hold water for easy passage. Suppositories lubricate the anus for easy stool passage.

c) Stool softeners such as docusate help mix fluid into stools to soften them.

d) Saline laxatives for example milk of magnesia draw fluid into the bowel from nearby tissue thereby softening the stools. e) The stimulant laxatives such as bisacodyl (Dulcolax) should not be used for more than a few days as they will cause the bowel to potentially lose tone.

C81 For gastrointestinal motility problems in PD:

- apply general measures for treating constipation. These include diet, laxatives, etc

- Reduce or discontinue drugs with anticholinergics activity. EFNS (GPP)

- Add domperidone. EFNS Level B

\section{Orthostatic Hypotension}

Orthostatic hypotension is seen in $30-58 \%$ of patients with Parkinson's disease. ${ }^{61}$ Important causes include: poor intake of fluids, side-effects of general medications such as antihypertensives, antidepressants, diuretics, other medical conditions such as cardiac dysfunction, diabetic neuropathy, PD dysautonomia, and side-effects of all PD medications especially dopamine agonists. A home blood pressure cuff for monitoring is often useful to document the severity and times of the orthostatic symptoms.

Initial general recommendations include adjustment of the medications if possible such as reducing antihypertensives and other medications causing hypotension, increasing daily water consumption, elevating the legs while sitting and getting up slowly especially early in the morning after sitting or lying for a period of time. This includes rising slowly after a meal. Aggravating factors such as large meals, alcohol, and exposure to excessive warmth need to be avoided. In the absence of any cardiovascular contraindication, salt intake can be increased by adding extra salt to meals or consuming salt using salt tablets or soup bouillon several times a day. However, it is estimated that up to eight grams of salt may be required to raise the blood pressure to make a clinical difference. Elevating the head of the bed or sleeping only partially reclined can help for early morning hypotension. Elastic stockings are often suggested but are difficult to get on and off and these stockings need to be fitted and patients need to be measured for pressures, generally around 30$40 \mathrm{~mm}$ of mercury. There is little evidence that their use alone without abdominal compression provides benefit.

Patients with orthostatic hypotension need to be warned that large meals could exacerbate this problem. Domperidone which can be given prior to every meal is used commonly in Canada for postprandial hypotension but good evidence for its effectiveness is lacking. ${ }^{38,62}$ If the hypotension is related to medications such as dopamine agonists then domperidone may be given one hour prior to the offending medication. Domperidone does not cause supine hypertension and does not cross the blood brain barrier. Mineralocorticoids such as fludrocortisone can be prescribed. In some patients and especially those that are immobile, this may cause pedal edema and therefore may not be the best first choice. Alpha-receptor agonists such as midodrine can often be a good choice and raises the orthostatic blood pressure but both fludrocortisone and midodrine can cause supine hypertension. 
C82 For orthostatic hypotension general measures would include:

- avoid aggravating factors such as large meals, alcohol, exposure to a warm environment and drugs known to cause orthostatic hypotension such as diuretics or antihypertensive drugs. Levodopa and dopamine agonists may also induce orthostatic hypotension.

- increase salt intake in symptomatic orthostatic hypotension.

- head-up tilt of the bed at night.

- wear elastic stockings.

- highlight postprandial effects. In some patients, hypotension occurs only postprandially. Warning the patient about this effect and taking frequent small meals may be helpful. EFNS (GPP)

C83 Drug therapy for orthostatic hypotension would include:

- add midodrine. EFNS Level A

- add fludrocortisone. EFNS (GPP)

\section{Erectile dysfunction}

This symptom is widespread in PD and reports suggest between $50-75 \%$ of men with PD will have this symptom. ${ }^{49}$ In addition to the dysautonomia caused by the PD, mood dysfunction, motor disability and side effects of medications may also contribute significantly. Good evidence for the use of sildenafil citrate (Viagra) exists but there is little reason not to think that similar medications like tadalafil (Cialis) and vardenafil (Levitra) would not also be effective.$^{38}$ Currently these drugs are not covered by provincial formularies for the purpose of treatment of erectile dysfunction in PD.

C84 For the treatment of erectile dysfunction in PD add sildenafil. EFNS Level A 


\section{Supplemental Tables}

\section{Supplemental Table 1: NICE - Classification of recommendations and evidence statements}

\begin{tabular}{|c|c|c|}
\hline Grade & Evidence & Type of Evidence \\
\hline A & $\begin{array}{l}\text { - At least one meta-analysis, systematic review, or } \\
\text { randomised controlled trial (RCT) that is rated as } 1^{++} \text {, } \\
\text { and is directly applicable to the target population, or } \\
\text { - A systematic review of RCTs or a body of evidence that } \\
\text { consists principally of studies rated as } 1+\text {, is directly } \\
\text { applicable to the target population and demonstrates } \\
\text { overall consistency of results, or } \\
\text { - Evidence drawn from a NICE technology appraisal }\end{array}$ & $\begin{array}{ll}1++ & \begin{array}{l}\text { High-quality meta-analysis (MA), systematic } \\
\text { reviews (SR) of randomized controlled trials } \\
\text { (RCTs), or RCTs with a very low risk of bias }\end{array} \\
1+\quad & \begin{array}{l}\text { Well-conducted MA, SR or RCTs, or RCTs } \\
\text { with a low risk of bias }\end{array}\end{array}$ \\
\hline $\mathrm{B}$ & $\begin{array}{l}\text { - A body of evidence that includes studies rated as } 2^{++} \text {, is } \\
\text { directly applicable to the target population and } \\
\text { demonstrates overall consistency of results, or } \\
\text { - Extrapolated evidence from studies rated as } 1^{++} \text {or } 1^{+}\end{array}$ & 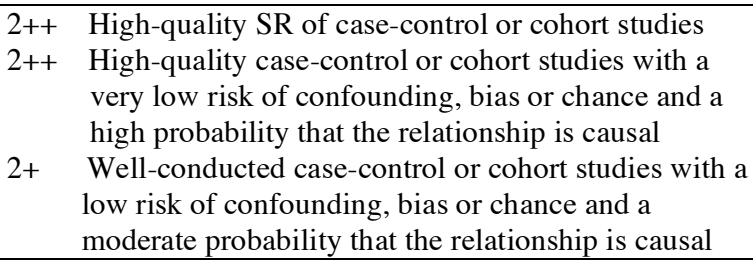 \\
\hline $\mathrm{C}$ & $\begin{array}{l}\text { - A body of evidence that includes studies rated as } 2^{+} \text {, is } \\
\text { directly applicable to the target population and } \\
\text { demonstrates overall consistency of results, or } \\
\text { - Extrapolated evidence from studies rated as } 2^{++}\end{array}$ & \\
\hline $\mathrm{D}$ & $\begin{array}{l}\text { - Evidence level } 3 \text { or } 4, \text { or } \\
\text { - Extrapolated evidence from studies rated as } 2^{+} \text {, or } \\
\text { - Formal consensus } \\
\end{array}$ & $\begin{array}{ll}3 & \begin{array}{l}\text { Non-analytic studies (for example case reports, case } \\
\text { series) }\end{array} \\
4 & \text { Expert opinion, formal consensus } \\
\end{array}$ \\
\hline $\mathrm{D}(\mathrm{GPP})$ & $\begin{array}{l}\text { A good practice point (GPP) is a recommendation for } \\
\text { best practice based on the experience of the Guideline } \\
\text { Development Group }\end{array}$ & \\
\hline
\end{tabular}

Supplemental Table 2: American Academy of Neurology - Classification of Evidence

\begin{tabular}{l|l}
\hline $\begin{array}{l}\text { Recommendation } \\
\text { grade }\end{array}$ & Evidence \\
\hline A & $\begin{array}{l}\text { Established as effective, ineffective, or harmful for the given condition in the specified } \\
\text { population. } \\
\text { Level A rating requires at least two consistent Class I studies }\end{array}$ \\
\hline $\mathrm{B}$ & $\begin{array}{l}\text { - Probably effective, ineffective, or harmful for the given condition in the specified population. } \\
\text { Level B rating requires at least one Class I study or at least two consistent Class II studies. }\end{array}$ \\
\hline $\mathrm{C}$ & $\begin{array}{l}\text { - Possibly effective, ineffective, or harmful for the given condition in the specified population. } \\
\text { Level C rating requires at least one Class II study or two consistent Class III studies. }\end{array}$ \\
\hline $\mathrm{U}$ & \begin{tabular}{l} 
Data inadequate or conflicting given current knowledge, treatment is unproven \\
\hline
\end{tabular}
\end{tabular}




\section{Supplemental Table 3: European Federation of Neurological Societies - Classification of Evidence}

\begin{tabular}{l|l}
\hline Recommendation grade & Evidence \\
\hline A & $\begin{array}{l}\text { established as effective, ineffective, or harmful, requires at least one } \\
\text { convincing class I study or at least two consistent, convincing class II } \\
\text { studies }\end{array}$ \\
\hline B & $\begin{array}{l}\text { probably effective, ineffective, or harmful, requires at least one convincing } \\
\text { class II study or overwhelming class III evidence }\end{array}$ \\
\hline C & $\begin{array}{l}\text { possibly effective, ineffective, or harmful rating, requires at least two } \\
\text { convincing class III studies }\end{array}$ \\
\hline GPP & $\begin{array}{l}\text { ("good practice point”) } \\
\text { where there is insufficient scientific evidence, a consensus statement }\end{array}$ \\
\hline
\end{tabular}

\section{Supplemental Table 4: Summary of the domain scores from the AGREE guideline instrument}

\begin{tabular}{|c|c|c|c|}
\hline & $\begin{array}{c}\text { Domain } 1 \\
\text { Scope and Purpose } \\
(\%)\end{array}$ & $\begin{array}{c}\text { Domain } 2 \\
\text { Stakeholder Involvement } \\
(\%)\end{array}$ & $\begin{array}{c}\text { Domain } 3 \\
\text { Rigour of Development } \\
(\%)\end{array}$ \\
\hline EFNS & 41 & 14 & 74 \\
\hline \multirow[t]{2}{*}{ NICE } & 89 & 77 & 79 \\
\hline & $\begin{array}{c}\text { Domain } 4 \\
\text { Clarity and Presentation } \\
(\%)\end{array}$ & $\begin{array}{c}\text { Domain } 5 \\
\text { Applicability } \\
\qquad \%)\end{array}$ & $\begin{array}{c}\text { Domain } 6 \\
\text { Editorial Independence } \\
(\%)\end{array}$ \\
\hline AAN & 72 & 78 & 83 \\
\hline EFNS & 53 & 41 & 55 \\
\hline
\end{tabular}

AAN - American Academy of Neurology; NICE - National Institute for Health and Clinical Excellence; EFNS - European Federation of Neurological Societies 


\section{Summary of Canadian Recommendations with References}

\section{Section 1}

\section{Communication}

C1 Communication with people with PD should be aimed towards empowering them to participate in the judgments and choices about their own care. NICE R1 Level D

C2 Discussions should be aimed at achieving a balance between the provision of honest, realistic information about the condition and the promotion of a feeling of optimism. NICE R2 Level D

C3 Because people with PD may develop impaired cognitive ability, a communication deficit and/or depression, they should be provided with: both oral and written communication throughout the course of the disease, which should be individually tailored and reinforced as necessary consistent communication from the professionals involved. NICE R3 Level D (GPP)

C4 Families and caregivers should be given information about the condition, their entitlements to care assessment and the support services available. NICE R4 Level D (GPP)

C5 People with PD should have a comprehensive care plan agreed between the individual, their family and/or caregivers and all healthcare providers NICE R5 Level D (GPP)

C6 People with PD should be offered an accessible point of contact with specialist services. NICE R6 Level D (GPP)

C7 Palliative care requirements of people with PD should be considered throughout all phases of the disease. NICE R82 Level D

C8 People with PD and their caregivers should be given the opportunity to discuss end-of life issues with appropriate healthcare professionals. NICE R83 Level D (GPP)

\section{Section 2}

\section{Diagnosis and Progression}

C9 PD should be suspected in people presenting with tremor, stiffness, slowness, balance problems and/or gait disorders. NICE R8 Level D (GPP)

C10 Determining the presence of the following clinical features in early stages of disease should be considered to distinguish PD from other parkinsonian syndromes: 1) falls at presentation and early in the disease course, 2) poor response to levodopa, 3) symmetry at onset, 4) rapid progression (to Hoehn and Yahr stage 3 in three years), 5) lack of tremor, and 6) dysautonomia (urinary urgency/incontinence and fecal incontinence, urinary retention requiring catheterization, persistent erectile failure or symptomatic orthostatic hypotension). AAN Diagnosis and Progression pg 972 Level B

C11 People with suspected PD should be referred quickly and untreated to a specialist with expertise in the differential diagnosis of this condition. NICE R11 Level B

C12 Clinicians should be encouraged to discuss with patients the possibility of tissue donation to a brain bank for purposes of diagnostic confirmation and research. NICE R10 Level D (GPP)

C13 There is insufficient evidence to determine whether levodopa and apomorphine challenge or olfaction testing have any advantage over the clinical diagnostic criteria of PD. AAN Diagnosis and Progression pg 972 Level U

C14 The following may not be useful in differentiating PD from other parkinsonian syndromes: GH stimulation with clonidine, electrooculography, and SPECT scanning. AAN Diagnosis and Progression pg 972 Level C

C15 There is insufficient evidence to support or refute the following as a means of distinguishing PD from other parkinsonian syndromes: urodynamics, autonomic testing, urethral or anal EMG, MRI, brain parenchyma sonography, and FDG PET. AAN Diagnosis and Progression pg 972 Level U

C16 In patients with newly diagnosed PD, older age at onset and rigidity/hypokinesia as an initial symptom should be used to predict more rapid rate of motor progression. AAN Diagnosis and Progression pg 974 Level B

C17 The presence of associated comorbidities (stroke, auditory deficits, and visual impairments), PIGD, and male sex may be used to predict faster rate of motor progression. AAN Diagnosis and Progression pg 974 Level C

C18 Tremor as a presenting symptom may be used to predict a more benign course and longer therapeutic benefit to levodopa. AAN Diagnosis and Progression pg 974 Level C

C19 Older age at onset and initial hypokinesia/rigidity should be used to predict earlier development of cognitive decline and dementia. AAN 2006 Diagnosis and Progression pg 974 Level B

C20 Older age of onset, dementia, and decreased dopamine responsiveness may be used to predict earlier nursing home placement as well as decreased survival. AAN Diagnosis and Progression pg 974 Level C 
C21 Vitamin E should not be used as a neuroprotective therapy for people with PD. NICE R22 Level A

C22 Co-enzyme Q10, dopamine agonists, monoamine oxidase B (MAO-B) inhibitors should not be used as a neuroprotective therapy for people with PD, except in the context of clinical trials. NICE R23, 24, 25 Level B

C23 There is insufficient evidence to support or refute the use of amantadine or thalamotomy for neuroprotection. AAN Neuroprotective strategies pg 979 Level U

C24 There is no long term evidence to recommend levodopa for neuroprotection. AAN Neuroprotective strategies page 979 Level U

\section{Section 3}

\section{Treatment - General considerations}

C25 Anti-parkinsonian medication should not be withdrawn abruptly or allowed to fail suddenly due to poor absorption (for example gastroenteritis, abdominal surgery) to avoid the potential for acute akinesia or neuroleptic malignant syndrome. NICE R51 Level D (GPP)

C26 The practice of withdrawing patients from their antiparkinsonian drugs (so-called 'drug holidays') to reduce motor complications should not be undertaken because of the risk of neuroleptic malignant syndrome. NICE R52 Level D (GPP)

C27 In view of the risks of sudden changes in anti-parkinsonian medication, people with PD who are admitted to hospital or care homes should have their medication: A) given at the appropriate times, which in some cases may mean allowing self-medication; B) adjusted by, or adjusted only after discussion with, a specialist in the management of PD. NICE R53 Level D (GPP)

C28 Clinicians should be aware of dopamine dysregulation syndrome (impulse control disorders), an uncommon disorder in which dopaminergic medication misuse is associated with abnormal behaviours, including hypersexuality, pathological gambling and stereotypic motor acts. This syndrome may be difficult to manage. NICE R54 Level D (GPP)

\section{Section 3A}

\section{Treatment - Pharmacological therapy for motor symptoms in early PD}

C29 It is not possible to identify a universal first-choice drug therapy for people with early PD. The choice of drug first prescribed should take into account:

- clinical and lifestyle characteristics

- patient preference, after the patient has been informed of the short- and long-term benefits and drawbacks of the drug classes. NICE R37 Level D (GPP)
C30 Levodopa may be used as a symptomatic treatment for people with early PD. NICE R26 Level A

C31 The dose of levodopa should be kept as low as possible to maintain good function in order to reduce the development of motor complications. NICE R27 Level A

C32 Modified-release levodopa preparations should not be used to delay the onset of motor complications in people with early PD. NICE R36 Level A

C33 Dopamine agonists may be used as a symptomatic treatment for people with early PD. NICE R28 Level A

C34 A dopamine agonist should be titrated to a clinically efficacious dose. If side effects prevent this, another agonist or a drug from another class should be used in its place. NICE R29 Level D (GPP)

C35 If an ergot-derived dopamine agonist is used, the patient should have a minimum of renal function tests, erythrocyte sedimentation rate (ESR) and chest radiograph performed before starting treatment, and annually thereafter. NICE R30 Level D (GPP)

C36 In view of the monitoring required with ergot-derived dopamine agonists, a non-ergot-derived agonist should be preferred in most cases. NICE R31 Level D (GPP)

C37 MAO-B inhibitors may be used as a symptomatic treatment for people with early PD. NICE R32 Level A

C38 Amantadine may be used as a treatment for people with early PD but should not be a drug of first choice. NICE R34 Level D (GPP)

C39 Anticholinergics may be used as a symptomatic treatment typically in young people with early PD and severe tremor, but should not be drugs of first choice due to limited efficacy and the propensity to cause neuropsychiatric side effects. NICE R35 Level B

C40 Beta-adrenergic antagonists may be used in the symptomatic treatment of selected people with postural tremor in PD, but should not be drugs of first choice. NICE R33 Level D (GPP)

\section{Section 3B}

Treatment - Pharmacological therapy for motor symptoms in later PD

C41 It is not possible to identify a universal first-choice adjuvant drug therapy for people with later PD. The choice of adjuvant drug first prescribed should take into account:

- clinical and lifestyle characteristics

- patient preference, after the patient has been informed of the short- and long-term benefits and drawbacks of the drug classes. NICE R50 Level D (GPP) 
C42 For patients with PD with motor fluctuations the available evidence suggests: Entacapone and rasagiline should be offered to reduce off time. AAN Motor Fluctuations pg 990 Level A

C43 Pramipexole and ropinirole should be considered to reduce off time. ***Tolcapone (hepatotoxicity) has limited availability in Canada and pergolide is not available in Canada secondary to its association with valvular fibrosis. ***AAN Motor Fluctuations pg 990 Level B

C44 Modified-release levodopa preparations may be used to reduce motor fluctuations in people with later $\mathrm{PD}$ but should not be drugs of first choice. NICE R38 Level B

C45 Amantadine may be considered for patients with PD with motor fluctuations in reducing dyskinesias. AAN Motor Fluctuations pg 991 Level C

\section{Section 3C}

\section{Treatment - Surgery}

C46 DBS of the STN may be considered as a treatment option in PD patients to improve motor function and to reduce motor fluctuations, dyskinesia, and medication usage. Patients need to be counseled regarding the risks and benefits of this procedure. AAN Motor Fluctuations pg 993 Level C

C47 Bilateral GPi stimulation may be used in people with PD who:

- have motor complications that are refractory to best medical treatment,

- are biologically fit with no clinically significant active comorbidity

- are levodopa responsive

- have no clinically significant active mental health problems, for example depression or dementia. NICE R56 Level D

C48 With the current evidence it is not possible to decide if the STN or GPi is the preferred target for DBS for people with $\mathrm{PD}$, or whether one form of surgery is more effective or safer than the other. In considering the type of surgery, account should be taken of:

- clinical and lifestyle characteristics of the person with PD

- patient preference after the patient has been informed of the potential benefits and drawbacks of the different surgical procedures. NICE R57 Level D

C49 Thalamic DBS may be considered as an option in people with PD who predominantly have severe disabling tremor and where STN stimulation cannot be performed. NICE R58 Level D

C50 Preoperative response to levodopa should be considered as a factor predictive of outcome after DBS of the STN. Motor Fluctuations pg 994 AAN Level B
C51 There is insufficient evidence to make any recommendations about factors predictive of improvement after DBS of the GPi or VIM nucleus of the thalamus in PD patients. AAN Motor Fluctuations pg 994 Level U

C52 Age and duration of PD may be considered as factors predictive of outcome after DBS of the STN. Younger patients with shorter disease durations may possibly have improvement greater than that of older patients with longer disease durations. AAN Motor Fluctuations pg 994 Level C

\section{Section 3D}

\section{Treatment - Other Treatment Options}

C53 People with PD should have regular access to the following:

- clinical monitoring and medication adjustment

- a continuing point of contact for support, including home visits, when appropriate

- a reliable source of information about clinical and social matters of concern to people with PD and their caregivers which may be provided by a Parkinson's disease nurse specialist. NICE R77 Level C

C54 Physical and exercise therapies should be available for people with PD. Particular consideration should be given to:

- gait re-education, improvement of balance and flexibility

- enhancement of aerobic capacity

- improvement of movement initiation

- improvement of functional independence, including mobility and activities of daily living

- provision of advice regarding safety in the home environment. NICE R78 Level B

C55 Occupational therapy should be available for people with PD. Particular consideration should be given to:

- maintenance of work and family roles, home care and leisure activities

- improvement and maintenance of transfers and mobility

- improvement of personal self-care activities, such as eating, drinking, washing and dressing

- environmental issues to improve safety and motor function

- cognitive assessment and appropriate intervention. NICE R80 Level D (GPP)

C56 Speech and language therapy should be available for people with PD. Particular consideration should be given to:

- improvement of vocal loudness and pitch range, including speech therapy programs such as Lee Silverman Voice Treatment (LSVT) NICE R81 Level B

- teaching strategies to optimize speech intelligibility NICE R81 Level D (GPP)

- ensuring an effective means of communication is maintained throughout the course of the disease, including use of assistive technologies NICE R81 Level D (GPP) 
- review and management to support safety and efficiency of swallowing and to minimize the risk of aspiration. NICE R81 Level D (GPP)

C57 There is insufficient evidence to support or refute the use of acupuncture, manual therapy, biofeedback or the Alexander technique in the treatment of PD. AAN Neuroprotective pg 981 Level U

\section{Section 4A}

Non-motor features of Parkinson's disease - Mental health

\section{Depression}

C58 Clinicians should have a low threshold for diagnosing depression in PD. NICE R59 Level D (GPP)

C59 Clinicians should be aware that there are difficulties in diagnosing mild depression in people with PD because the clinical features of depression overlap with the motor features of PD. NICE R60 Level D (GPP)

C60 The management of depression in people with PD should be tailored to the individual, in particular, to their co-existing therapy. NICE R61 Level D (GPP)

C61 Amitriptyline may be considered in the treatment of depression associated with PD AAN Depression, Psychosis and Dementia pg 1000 Level C

\section{Psychotic symptoms}

C62 All people with PD and psychosis should receive a general medical evaluation and treatment for any precipitating condition. NICE R62 Level D (GPP)

C63 Reduce polypharmacy. Reduce/stop anticholinergic antidepressants, reduce/stop anxiolytics/sedatives. EFNS Part II page 1192 (GPP)

C64 Consideration should be given to withdrawing gradually antiparkinsonian medication that might have triggered psychosis in people with PD. NICE R63 Level D (GPP)

C65 Reduce antiparkinsonian drugs. Stop anticholinergics, stop amantadine, reduce/stop dopamine agonists, reduce/stop MAO-B and COMT inhibitors, lastly reduce levodopa. Stopping antiparkinsonian drugs can be at the cost of worsening motor symptoms. EFNS Part II page 1192 Level $\mathrm{D}(\mathrm{GPP})$

C66 Mild psychotic symptoms in people with PD may not need to be actively treated if they are well tolerated by the patient and caregiver. NICE R64 Level D (GPP)

C67 Typical antipsychotic drugs (such as phenothiazines and butyrophenones) should not be used in people with PD because they exacerbate the motor features of the condition. NICE R65 Level D (GPP)

C68 For patients with PD and psychosis, olanzapine should NOT be routinely considered. AAN Depression, Psychosis and Dementia pg 1000 Level B

C69 Clozapine may be used in the treatment of psychotic symptoms in $\mathrm{PD}$, but registration with a mandatory monitoring scheme is required. It is recognized that few specialists caring for people with PD have experience with clozapine. NICE R67 Level B

C70 For patients with PD and psychosis, quetiapine may be considered. AAN Depression, Psychosis and Dementia pg 1000 Level C

\section{Dementia}

C71 Discontinue potential aggravators.

- Anticholinergics. EFNS Part II pg 1192 Level B

- amantadine, tricyclic antidepressants, benzodiazepines, tolterodine and oxybutynin. EFNS Part II pg 1192 Level C

C72 Donepezil should be considered for the treatment of dementia in PD. AAN Depression, Psychosis and Dementia pg 1001 Level B

C73 Rivastigmine should be considered for the treatment of dementia in PD or DLB. AAN Depression, Psychosis and Dementia pg 1000 Level B

\section{Section 4B}

\section{Non-motor features of Parkinson's disease - Sleep}

C74 A full sleep history should be taken from people with PD who report sleep disturbance. NICE R69 Level D (GPP)

C75 Good sleep hygiene should be advised in people with PD with any sleep disturbance and includes:

- avoidance of stimulants (for example, coffee, tea, caffeine) in the evening

- establishment of a regular pattern of sleep

- comfortable bedding and temperature

- provision of assistive devices, such as a bed lever or rails to aid with moving and turning, allowing the person to get more comfortable restriction of daytime siestas advice about taking regular and appropriate exercise to induce better sleep

- a review of all medication and avoidance of any drugs that may affect sleep or alertness, or may interact with other medication (for example, selegiline, antihistamines, $\mathrm{H} 2$ antagonists, antipsychotics and sedatives). NICE R70 Level D (GPP)

C76 Care should be taken to identify and manage restless legs syndrome (RLS) and rapid eye movement (REM) sleep 
behaviour disorder in people with PD and sleep disturbance. NICE R71 Level D (GPP)

C77 People with PD who have sudden onset of sleep should be advised not to drive and to consider any occupational hazards. Attempts should be made to adjust their medication to reduce its occurrence. NICE R72 Level D (GPP)

C78 Modafinil may be considered for daytime hypersomnolence in people with PD. NICE R73 Level D (GPP)

\section{Section 4C \\ Non-motor features of Parkinson's disease - autonomic dysfunction}

C79 People with PD should be treated appropriately for the following autonomic disturbances:

- urinary dysfunction

- weight loss

- dysphagia

- constipation

- erectile dysfunction

- orthostatic hypotension

- excessive sweating

- sialorrhoea

NICE R76 Level D (GPP)

C80 General measures for treating urinary urgency and incontinence include: avoiding coffee before bedtime, limit water ingestion before bedtime, etc.

- Add peripherally acting anticholinergic drugs. EFNS Part II pg 1194 Level GPP

C81 For gastrointestinal motility problems in PD

- apply general measures for treating constipation. These include diet, laxatives, etc

- Reduce or discontinue drugs with anticholinergics activity. EFNS Part II pg 1195 Level GPP

- Add domperidone. EFNS Part II pg 1195 Level B

C82 For orthostatic hypotension general measures would include:

- avoid aggravating factors such as large meals, alcohol, exposure to a warm environment and drugs known to cause orthostatic hypotension such as diuretics or antihypertensive drugs. Levodopa and dopamine agonists may also induce orthostatic hypotension.

- increase salt intake in symptomatic orthostatic hypotension.

- head-up tilt of the bed at night.

- wear elastic stockings.

- highlight postprandial effects. In some patients, hypotension occurs only postprandially. Warning the patient about this effect and taking frequent small meals may be helpful. EFNS Part II pg 1194 Level GPP
C83 Drug therapy for orthostatic hypotension would include:

- add midodrine. EFNS Part II pg 1194 Level A

- add fludrocortisone. EFNS Part II pg 1194 Level GPP

C84 For the treatment of erectile dysfunction in PD add sildenafil - EFNS Part II pg 1195 Level A

\section{REFERENCES FOR GUIDELINES USED}

- Practice parameter: diagnosis and prognosis of new onset Parkinson disease (an evidence-based review). Report of the Quality Standards Subcommittee of the American Academy of Neurology. Neurology. 2006;66:968-75 .

- Practice parameter: evaluation and treatment of depression, psychosis, and dementia in Parkinson disease (an evidence-based review). Report of the Quality Standards Subcommittee of the American Academy of Neurology. Neurology. 2006;66:996-1002.

- Practice Parameter: treatment of Parkinson disease with motor fluctuations and dyskinesia (an evidence-based review): report of the Quality Standards Subcommittee of the American Academy of Neurology. Neurology. 2006;66:983-95.

- Practice parameter: neuroprotective strategies and alternative therapies for Parkinson disease (an evidence-based review). Report of the Quality Standards Subcommittee of the American Academy of Neurology. Neurology. 2006;66:976-82.

- Practice parameter: initiation of treatment for Parkinson's disease: an evidence-based review. Neurology. 2002;58:11-17.

- Review of the therapeutic management of Parkinson's disease. Report of a joint task force of the European Federation of Neurological Societies and the Movement Disorder SocietyEuropean Section. Part I: early (uncomplicated) Parkinson's disease. European Journal of Neurology. 2006;13:1170-85.

- Review of the therapeutic management of Parkinson's disease. Report of a joint task force of the European Federation of Neurological Societies (EFNS) and the Movement Disorder SocietyEuropean Section (MDS-ES). Part II: late (complicated) Parkinson's disease. European Journal of Neurology. 2006;13: 1186-202.

- National Institute for Health and Clinical Excellence - Parkinson's disease: diagnosis and management in primary and secondary care. June 2006.

- http://www.nice.org.uk/nicemedia/pdf/cg035niceguideline.pdf.

\section{SPECIFIC Chapter ReFERENCES}

1. Gibb WR, Lees AJ. The relevance of the Lewy body to the pathogenesis of idiopathic Parkinson's disease. J Neurol Neurosurg Psychiatry. 1988;51(6):745-52.

2. Schrag A, Ben-Shlomo Y, Quinn N. How valid is the clinical diagnosis of Parkinson's disease in the community? J Neurol Neurosurg Psychiatry. 2002;73(5):529-34.

3. Olanow CW, Rascol O, Hauser R, et al. A double-blind, delayedstart trial of rasagiline in Parkinson's disease. N Engl J Med. 2009;361 (13):1268-78.

4. Schapira AH, Obeso J. Timing of treatment initiation in Parkinson's disease: a need for reappraisal? Ann Neurol. 2006;59(3):559-62.

5. Stocchi F, Rascol O, Kieburtz K, et al. Initiating levodopa/carbidopa therapy with and without entacapone in early Parkinson disease: the STRIDE-PD study. Ann Neurol. 2010;68(1): 18-27.

6. Weintraub D, Koester J, Potenza MN, et al. Impulse control disorders in Parkinson disease: a cross-sectional study of 3090 patients. Arch Neurol. 2010;67(5):589-95.

7. Hassan A, Bower JH, Kumar N, et al. Dopamine agonist-triggered pathological behaviors: surveillance in the PD clinic reveals high frequencies. Parkinsonism Relat Disord. 2011;17(4):260-4.

8. Moro E, Lozano AM, Pollak P, et al. Long-term results of a multicenter study on subthalamic and pallidal stimulation in Parkinson's disease. Mov Disord. 2010;25(5):578-86.

9. Hamani C, Lozano AM. Hardware-related complications of deep brain stimulation: a review of the published literature. Stereotact Funct Neurosurg. 2006;84(5-6):248-51. 
10. Hariz MI, Rehncrona S, Quinn NP, Speelman JD, Wensing C. Multicenter study on deep brain stimulation in Parkinson's disease: an independent assessment of reported adverse events at 4 years. Mov Disord. 2008;23(3):416-21.

11. Deuschl G, Schade-Brittinger C, Krack P, et al. A randomized trial of deep-brain stimulation for Parkinson's disease. N Engl J Med. 2006;355(9):896-908

12. Weaver FM, Follett K, Stern M, et al. Bilateral deep brain stimulation vs best medical therapy for patients with advanced Parkinson disease: a randomized controlled trial. JAMA. 2009; 301(1):63-73.

13. Pahwa R, Lyons KE, Wilkinson SB, et al. Long-term evaluation of deep brain stimulation of the thalamus. J Neurosurg. 2006;104(4): 506-12.

14. Hariz MI, Krack P, Alesch F, et al. Multicentre European study of thalamic stimulation for parkinsonian tremor: a 6 year follow-up. J Neurol Neurosurg Psychiatry. 2008;79(6):694-9.

15. Derost PP, Ouchchane L, Morand D, et al. Is DBS-STN appropriate to treat severe Parkinson disease in an elderly population? Neurology. 2007;68(17):1345-55.

16. Schupbach WM, Maltete D, Houeto JL, et al. Neurosurgery at an earlier stage of Parkinson disease: a randomized, controlled trial. Neurology. 2007;68(4):267-71

17. Ravina B, Camicioli R, Como PG, et al. The impact of depressive symptoms in early Parkinson disease. Neurology. 2007;69(4): 342-7.

18. Menza M, Dobkin RD, Marin $\mathrm{H}$, et al. A controlled trial of antidepressants in patients with Parkinson disease and depression. Neurology. 2009;72(10):886-92.

19. Antonini A, Tesei S, Zecchinelli A, et al. Randomized study of sertraline and low-dose amitriptyline in patients with Parkinson's disease and depression: effect on quality of life. Mov Disord. 2006;21(8):1119-22.

20. Devos D, Dujardin K, Poirot I, et al. Comparison of desipramine and citalopram treatments for depression in Parkinson's disease: a double-blind, randomized, placebo-controlled study. Mov Disord. 2008;23(6):850-7.

21. Weintraub D, Mavandadi S, Mamikonyan E, et al. Atomoxetine for depression and other neuropsychiatric symptoms in Parkinson disease. Neurology. 2010;75(5):448-55

22. Barone P, Poewe W, Albrecht S, et al. Pramipexole for the treatment of depressive symptoms in patients with Parkinson's disease: a randomised, double-blind, placebo-controlled trial. Lancet Neurol. 2010;9(6):573-80.

23. Friedman JH. Parkinson's disease psychosis 2010: a review article. Parkinsonism Relat Disord. 2010;16(9):553-60.

24. Rabey JM, Prokhorov T, Miniovitz A, Dobronevsky E, Klein C. Effect of quetiapine in psychotic Parkinson's disease patients: a double-blind labeled study of 3 months' duration. Mov Disord. 2007;22(3):313-8.

25. Shotbolt P, Samuel M, Fox C, David AS. A randomized controlled trial of quetiapine for psychosis in Parkinson's disease. Neuropsychiatr Dis Treat. 2009;5:327-32.

26. Merims D, Balas M, Peretz C, Shabtai H, Giladi N. Rater-blinded, prospective comparison: quetiapine versus clozapine for Parkinson's disease psychosis. Clin Neuropharmacol. 2006;29(6): 331-7.

27. Hely MA, Reid WG, Adena MA, Halliday GM, Morris JG. The Sydney multicenter study of Parkinson's disease: the inevitability of dementia at 20 years. Mov Disord. 2008;23(6):837-44.

28. Aarsland D, Ballard C, Walker Z, et al. Memantine in patients with Parkinson's disease dementia or dementia with Lewy bodies: a double-blind, placebo-controlled, multicentre trial. Lancet Neurol. 2009;8(7):613-8.

29. Grace J, Amick MM, Friedman JH. A double-blind comparison of galantamine hydrobromide ER and placebo in Parkinson disease. J Neurol Neurosurg Psychiatry. 2009;80(1):18-23.

30. Emre M, Tsolaki M, Bonuccelli U, et al. Memantine for patients with Parkinson's disease dementia or dementia with Lewy bodies: a randomised, double-blind, placebo-controlled trial. Lancet Neurol. 2010;9(10):969-77.
31. Dauvilliers Y. Insomnia in patients with neurodegenerative conditions. Sleep Med. 2007;8 Suppl 4:S27-34.

32. Montgomery P, Dennis J. A systematic review of nonpharmacological therapies for sleep problems in later life. Sleep Med Rev. 2004;8(1):47-62.

33. Dowling GA, Mastick J, Colling E, Carter JH, Singer CM, Aminoff MJ. Melatonin for sleep disturbances in Parkinson's disease. Sleep Med. 2005;6(5):459-66.

34. Medeiros CA, Carvalhedo de Bruin PF, Lopes LA, Magalhaes MC, de Lourdes Seabra M, de Bruin VM. Effect of exogenous melatonin on sleep and motor dysfunction in Parkinson's disease. A randomized, double blind, placebo-controlled study. J Neurol. 2007;254(4):459-64

35. Leeman AL, O'Neill CJ, Nicholson PW, et al. Parkinson's disease in the elderly: response to and optimal spacing of night time dosing with levodopa. Br J Clin Pharmacol. 1987;24(5):637-43.

36. Stocchi F, Barbato L, Nordera G, Berardelli A, Ruggieri S. Sleep disorders in Parkinson's disease. J Neurol. 1998;245 Suppl 1: S15-8

37. Menza M, Dobkin RD, Marin H, et al. Treatment of insomnia in Parkinson's disease: a controlled trial of eszopiclone and placebo. Mov Disord. 2010;25(11):1708-14.

38. Zesiewicz TA, Sullivan KL, Arnulf I, et al. Practice Parameter: treatment of nonmotor symptoms of Parkinson disease: report of the Quality Standards Subcommittee of the American Academy of Neurology. Neurology. 2010;74(11):924-31.

39. Gagnon JF, Postuma RB, Mazza S, Doyon J, Montplaisir J. Rapideye-movement sleep behaviour disorder and neurodegenerative diseases. Lancet Neurol. 2006;5(5):424-32.

40. Gagnon JF, Postuma RB, Montplaisir J. Update on the pharmacology of REM sleep behavior disorder. Neurology. 2006;67(5):742-7

41. Aurora RN, Zak RS, Maganti RK, et al. Best practice guide for the treatment of REM sleep behavior disorder (RBD). J Clin Sleep Med. 2010;6(1):85-95.

42. Ferini-Strambi L, Manconi M. Treatment of restless legs syndrome. Parkinsonism Relat Disord. 2009;15 Suppl 4:S65-70.

43. Arnulf I. Excessive daytime sleepiness in parkinsonism. Sleep Med Rev. 2005;9(3):185-200.

44. Cochen De Cock V, Abouda M, Leu S, et al. Is obstructive sleep apnea a problem in Parkinson's disease? Sleep Med. 2010;11(3): 247-52.

45. Adler CH, Caviness JN, Hentz JG, Lind M, Tiede J. Randomized trial of modafinil for treating subjective daytime sleepiness in patients with Parkinson's disease. Mov Disord. 2003;18(3): 287-93.

46. Ondo WG, Fayle R, Atassi F, Jankovic J. Modafinil for daytime somnolence in Parkinson's disease: double blind, placebo controlled parallel trial. J Neurol Neurosurg Psychiatry. 2005;76 (12):1636-9.

47. Hogl B, Saletu M, Brandauer E, et al. Modafinil for the treatment of daytime sleepiness in Parkinson's disease: a double-blind, randomized, crossover, placebo-controlled polygraphic trial. Sleep. 2002;25(8):905-9.

48. Magerkurth C, Schnitzer R, Braune S. Symptoms of autonomic failure in Parkinson's disease: prevalence and impact on daily life. Clin Auton Res. 2005;15(2):76-82.

49. Chaudhuri KR, Healy DG, Schapira AH. Non-motor symptoms of Parkinson's disease: diagnosis and management. Lancet Neurol. 2006;5(3):235-45

50. Bachmann CG, Trenkwalder C. Body weight in patients with Parkinson's disease. Mov Disord. 2006;21(11):1824-30.

51. Barichella M, Cereda E, Pezzoli G. Major nutritional issues in the management of Parkinson's disease. Mov Disord. 2009;24(13): 1881-92.

52. Naumann M, So Y, Argoff CE, et al. Assessment: Botulinum neurotoxin in the treatment of autonomic disorders and pain (an evidence-based review): report of the Therapeutics and Technology Assessment Subcommittee of the American Academy of Neurology. Neurology. 2008;70(19):1707-14.

53. Hyson HC, Johnson AM, Jog MS. Sublingual atropine for sialorrhea secondary to parkinsonism: a pilot study. Mov Disord. 2002;17 (6):1318-20. 
54. Molloy L. Treatment of sialorrhoea in patients with Parkinson's disease: best current evidence. Curr Opin Neurol. 2007;20(4): 493-8.

55. Thomsen TR, Galpern WR, Asante A, Arenovich T, Fox SH. Ipratropium bromide spray as treatment for sialorrhea in Parkinson's disease. Mov Disord. 2007;22(15):2268-73.

56. Simuni T, Sethi K. Nonmotor manifestations of Parkinson's disease. Ann Neurol. 2008;64 Suppl 2:S65-80.

57. Chancellor MB, Anderson RU, Boone TB. Pharmacotherapy for neurogenic detrusor overactivity. Am J Phys Med Rehabil. 2006;85(6):536-45.

58. Mostile G, Jankovic J. Treatment of dysautonomia associated with Parkinson's disease. Parkinsonism Relat Disord. 2009;15 Suppl 3:S224-32.
59. Santos JC, Telo ER. Solifenacin: scientific evidence in the treatment of overactive bladder. Arch Esp Urol. 2010;63(3):197213.

60. Abbott RD, Ross GW, Petrovitch H, et al. Bowel movement frequency in late-life and incidental Lewy bodies. Mov Disord. 2007;22(11):1581-6.

61. Goldstein DS. Orthostatic hypotension as an early finding in Parkinson's disease. Clin Auton Res. 2006;16(1):46-54.

62. Schoffer KL, Henderson RD, O'Maley K, O'Sullivan JD. Nonpharmacological treatment, fludrocortisone, and domperidone for orthostatic hypotension in Parkinson's disease. Mov Disord. 2007;22(11):1543-9. 
https://doi.org/10.1017/S031716710001516X Published online by Cambridge University Press 


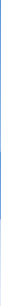

